\title{
Contribution à l'étude de la variabilité biologique et biochimique du pin à crochets (Pinus uncinata Ram.) dans les Pyrénées
}

\author{
R. CANTEGREL * \\ Centre de Biologie des Ecosystèmes d'altitude \\ Université de Pan et des Pays de l'Adour, F 64000 Paı**
}

\section{Résumé}

Le pin à crochets (Pinus uncinata Ramond) présente un large éventail de formes, aussi bien au niveau individuel qu'entre populations. Dans les Pyrénées occidentales, le hiatus morphologique est particulièrement accentué entre le massif calcaire d'Anie, aux arbres souffreteux. et le massif granitique de Néouvielle, d'aspect beaucoup plus vigoureux. La comparaison de ces deux provenances s'effectue in situ sur la base d'une étude biosystématique mettant en jeu un grand rombre de caractères, qu'ils soient morphologiques (pollen, aiguille, cône, graine, plantule) ou biochimiques (terpènes des tissus corticaux).

L'analyse de covariance au sein de chaque population permet d'éliminer de l'étude taxonomique d'une part les caractères directement sensibles aux effets de l'environnement, et d'autre part les données redondantes. Les caractères de cône, d'aiguille et de profil terpénique, une fois épurés des effets d'âge ou de vigueur des arbres-échantillons, font. l'objet d'une analyse discriminante renforçant la divergence entre les deux populations déjà obtenue lors de l'étude séparée des caractères.

La fiabilité des caractères terpéniques en tant que marqueurs génétiques amène à considérer Anie et Néouvielle comme deux taxons distincts de pin à crochets dont le plus occidental (Anie) doit probablement sa variabilité phénotypique relativement réduite à sa position marginale par rapport à l'aire pyrénéenne de Pinus uncinata.

\section{Introduction}

Le complexe des pins de montagne (Pinus montana Mill.) peuple les montagnes d'Europe depuis les Monts Ibériques jusqu'aux Carpates, en de multiples populations d'altitude ou de tourbière. Alors que la zone centrale et orientale de l'aire comporte en majorité l'espèce rampante (Pinus mughus Scop.), le pin à crochets (Pinus uncinata Ram.) domine à l'Ouest et trouve dans les Pyrénées catalanes son optimum bioclimatique (GAUSSEN, 1926). \footnotetext{
Girons.

* Adresse actuelle : Centre O.N.F. de Saint-Girons, 5, place François-Camel, F 09200 Saint-

*: Le présent mémoire condense les principaux résultats d'une thèse de $3^{\text {c }}$ cycle menée à bien en 1982 grâce à la collaboration :

- du Laboratoire de Physiologie cellulaire végétale, Université de Bordeaux I, F 33405 Talence;

- et du Laboratoire d'Amélioration des Arbres forestiers, I.N.R.A.-Bordeaux, F 33610 Cestas.
} 
Moins connus sont les massifs pyrénéens centraux et occidentaux où le polymorphisme du pin, tant au niveau de la population quau niveau individuel, atteint un degré particulièrement élevé dans un environnement climatique et édaphique rigoureux et très hétérogène (Dindali:Tcile, 1973).

Dans ces conditions, l'explication de la variabilité biologique de Pinus uncinata à partir d'une étude biosystématique des populations naturelles, doit intégrer aussi bien l'amplitude éco-éthologique du pin que sa diversité génétique.

\section{Matériel et méthode}

\subsection{Echantillonnage}

L'absence de dispositif expérimental autorisant des comparaisons morphologiques ou physiologiques de provenances en milieu uniforme, oblige à sélectionner un grand nombre d'attributs végétatifs ou génératifs dont la caractérisation s'effectue in situ ou au laboratoire, et à apprécier les principaux paramètres environnementaux. En contrepartie, l'analyse de la variation géographique se trouve essentiellement concentrée sur deux pincraies distantes de $80 \mathrm{~km}$ dans les Pyrénées nord-occidentales entre lesquelles les hiatus morphologiques et écologiques apparaissent privilégiés :

- le massif karstique d'Anie, recevant de plein fouet les masses d'air atlantiques, où la forêt très claire et d'aspect souffreteux colonise entre 1600 et $2100 \mathrm{~m}$ les horts calcaires profondément lapiazés et dénudés;

- le massif granitique de Néouvielle, en position interne, jouissant d'un climat remarquablement lumineux (CHOUARD, 1938), où les peuplements plus denses et vigoureux s'étagent de 1800 à $2600 \mathrm{~m}$.

Au sein de chacune de ces populations on désigne selon un transect altitudinal une quarantaine d'arbres-échantillons servant de base aux comparaisons morphologiques et biochimiques.

A titre de référence, quelques sondages intéressent des populations centrales et orientales : massif des Encantats au versant Sud des Pyrénécs, tourbière de Pinet à $880 \mathrm{~m}$ d'altitude sur le plateau de Sault, forêts du Puigmal et du Capcir (fig. 1).

\subsection{Matériel mesuré}

On cherche à diversifier au maximum les éléments analysés et à multiplier les mesures afin de sélectionner les caractères les plus discriminants (labl. 1).

\subsection{Observations}

Le matériel prélevé sur chaque arbre-échantillon est emballé dans une poche en nylon portant la référence de l'arbre parental, de façon à toujours être en mcsure de comparer la variabilité intra-arbres à celle existant entre les individus de la même population. Seuls les pollens d'Anie et de Néouvielle et les cônes issus des Encantats n'ont pu être récoltés selon ce protocole, et on ne dispose pour ce matéricl que de moyennes de populations. 


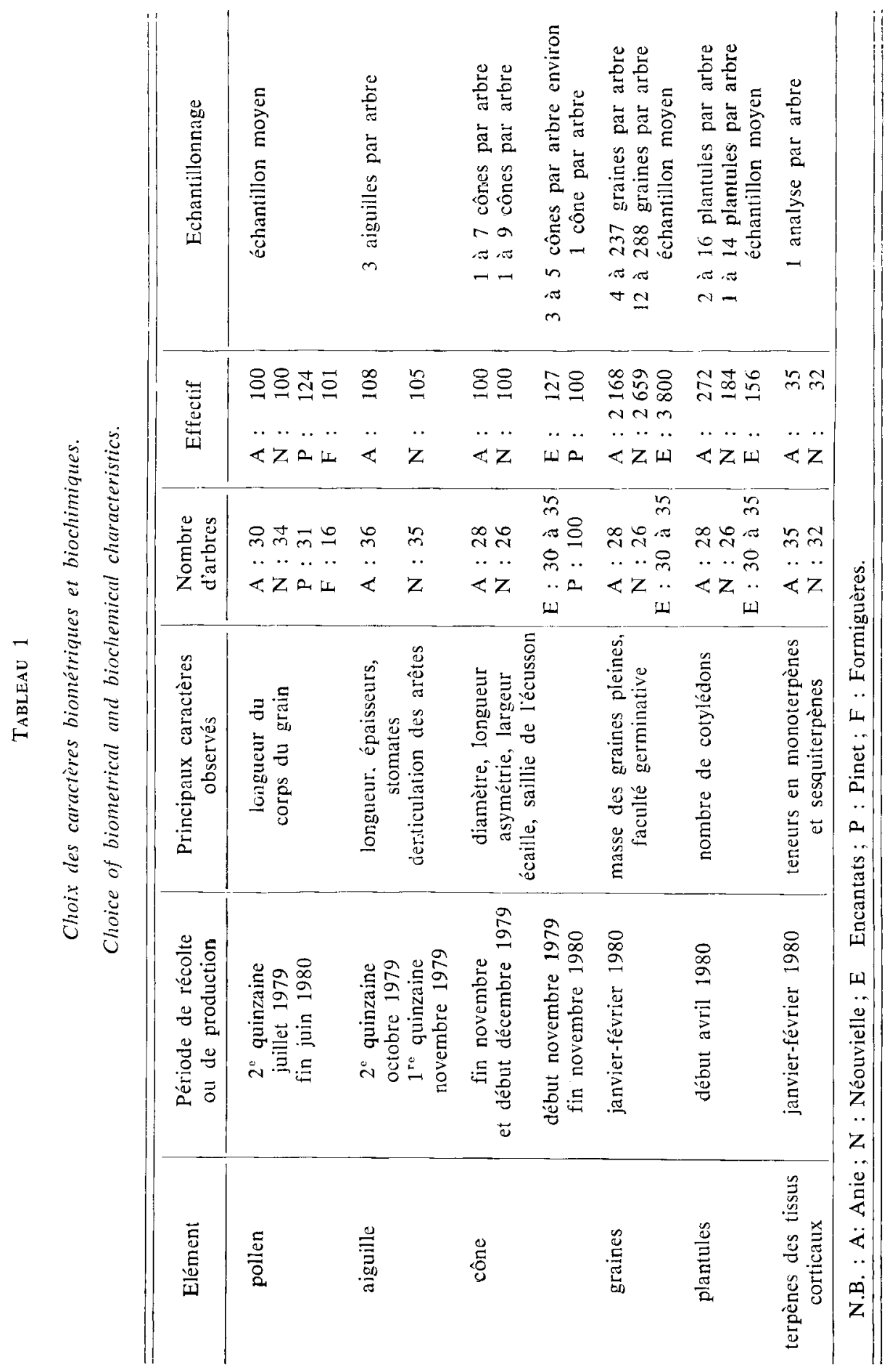




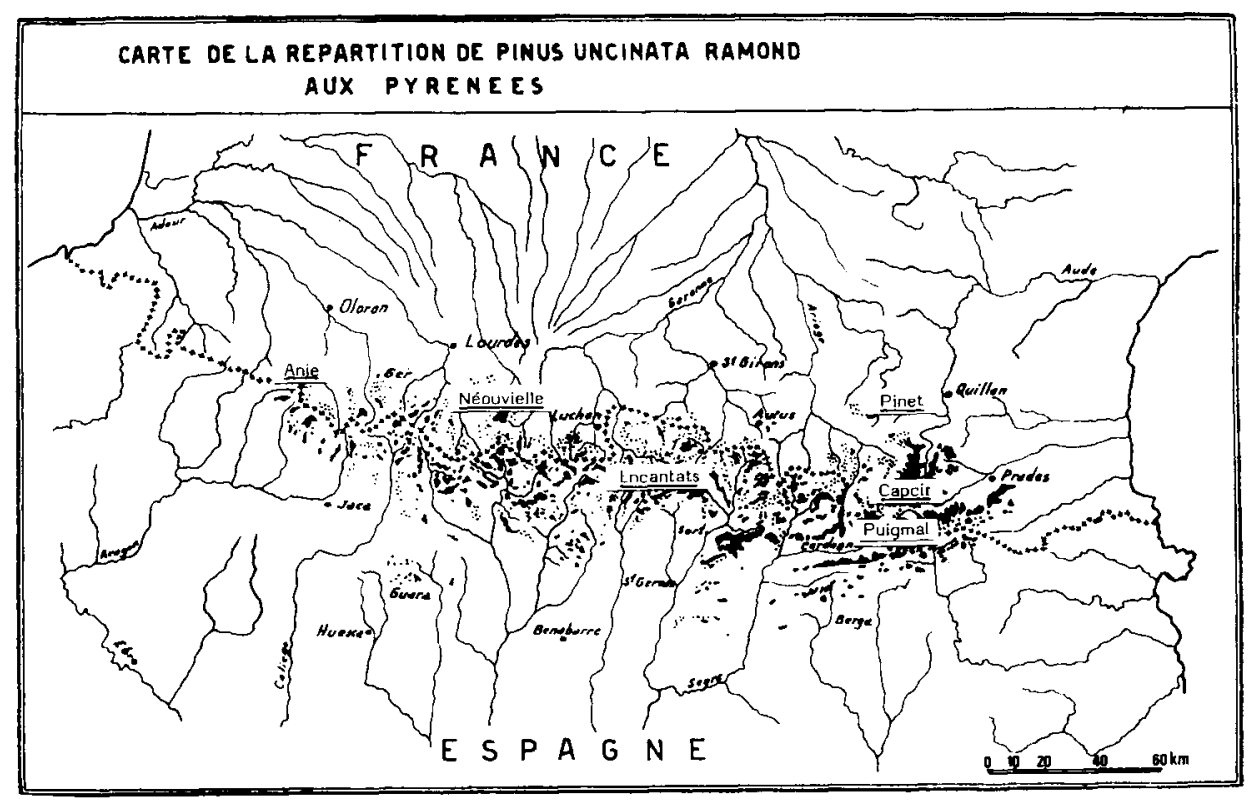

FIG. 1

Carte de la répartition de Pinus uncinata Ramond anx Pyrénées et populations de référence. Pyrenean area of Pinus uncinata Ramond and sampling populations.

Il se peut que la variation inter-annuelle des caractéristiques de divers organes, en particulier des pollens et des graines, ne soit pas négligeable vis-à-vis des aléas climatiques, et il conviendrait d'effectuer d'autres campagnes de mesures pour éprouver la stabilité de ces critères. En revanche, la sćcurité des résultats obtenus à partirdes cônes, des aiguilles et des terpènes paraît satisfaisante dans les conditions expérimentales retenues ici. Ainsi le cône mûr a-t-il subi également les conditions climatiques des deux années précédentes et le prélèvement des aiguilles et des lissus corticaux s'est-il effectué sur les trois derniers verticilles de façon à tamponner l'effet année.

Pour plus de sûreté, l'analyse discriminante développée au paragraphe 7 concerne uniquement ces trois groupes de caractères (cônes, aiguilles, terpènes).

\section{Le contexte écologique et dynamique}

\subsection{Les phytocénoses en présence}

L'étude phyto-écologique d’Anie et de Néouvielle montre que "l'étage " du pin à crochets n'est pas homogène et recouvre des entités écobiocénotiques bien typées, spécialement la ceinture altimontaine à sorbiers et bouleaux (Dendaletche, 1973) où le peuplement acquiert son maximum de densité, surmontée par le subalpin arboré (fig. 2). 


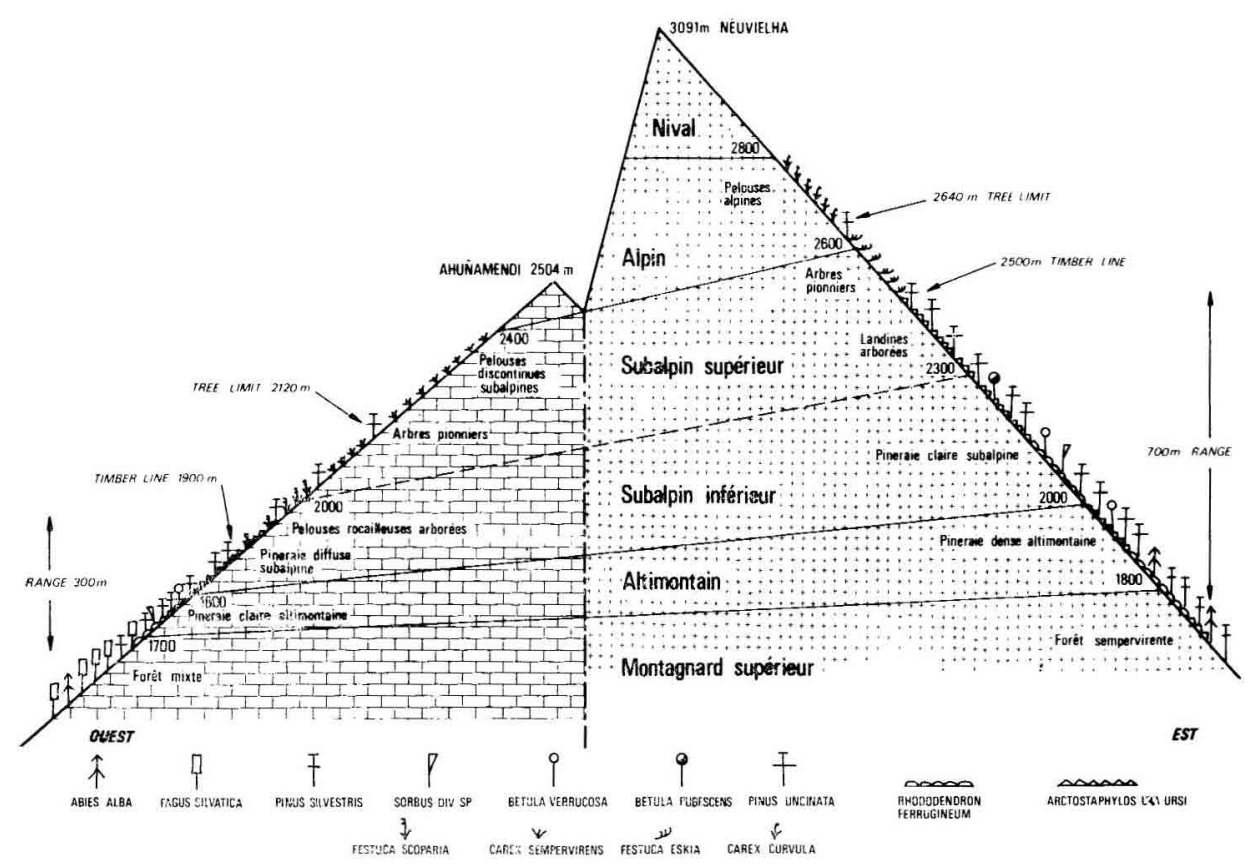

FIG. 2

Modalités de peuzlement altitudinal du pin à crochets dans les Pyrénées nord-occidentales (massifs d'Anie et de Néouvielle).

Altitudinal colonization of mountain pine in north-western Pyrenees

(Anie and Neouvielle stands).

Fioristiquement on peut caractẻriser les deux massits par deux ensembles vicariants typiques dans les Pyrénées occidentales :

- la pineraie karstique d'Anie à Arctostaphylos uva-ursi et Festuca scoparia; eskia.

- la pineraie cristalline de Néouvielle à Rhododendron ferrugineum et Festuca

Les principaux groupements végétaux où intervient Pinus uncinata à l'intérieur de ces deux grands ensembles se caractérisent par leur mosaïque, notamment sur substrat calcaire où les facteurs micro-stationnels sont déterminants (fentes karstiques, figures de dissolution, facettes d'exposition, végétaux acidifiants, ...).

On distingue schématiquement en Anie :

- La landine hygrophile de Rhododendron ferrugineum et Vaccinium myrtillus, en ombrée, avec Salix pyrenaica, Pulsatilla alpina, Rhamnus alpina, Geranium cinereum, Aquilegia pyrenaica, Bartschia alpina, et des éléments plus acidophiles : Arbutus alpina, Vaccinium uliginosum, Empetrum hermaphroditum...

- La dryadaie : landine hygro-héliophile de Dryas octopetala, en soulane et replat ensoleillé, avec Hypericum nummularium, Viola biflora, Betonica alopecuros, 
Horminum pyrenaicum. Dans les fentes du karst on découvre : Euphorbia chamaebuxus, Androsace villosa, Asperula hirta, Lonicera pyrenaica...

- L'arctostaphylaie : landine hélio-thermophile d'Arctostaphylos ura-ursi. Coloneaster integerrima, Amelanchier ovalis, Iminiperus nama, et le groupement rupicole composé de Globularia nana. Helianthenam canum. Thymus serpillum, Teucriam pyrenaicum...

- La pelouse rocailleuse de Sesleria coerulea et Carex sempervirens, et les îlots de Festuca scoparia. I. spadicea. Avena montana.

En Néouvielle :

- Les éboulis granitiques avec une abondance de Piéridophytes : Asplenium septentrionale, Aspidium lonchitis, Cystopleris fragilis, Polystichum filix-mas, Allosurus crispus, Dryopteris phegopteris, Lycopodium alpinum, et un lot d'arbrisseaux parmi lesquels : Rhamnus alpina. Cotoneaster integerrima, Ribes petraeum, Rosa glauca...

- La rhodoraic, aux ombrées : Rhododendron ferrugineum, Vacinium myrtillus, V. uliginosum, Homogyne alpina, Empetrum hermaphroditum, Hylocomium splendens, Cetraria islandica, Rosa pendalina...

- L'arctostaphylaie, aux soulanes : Aretostaphylos una-ursi, Juniperus nana. Calluna valgaris.

- La nardaie, pelouse généralement cantonnée aux zones concaves à forte accumulation nivale, rélractaire au pin à crochets : Nardus stricta. Solinum pyreneum. Festuca rubra, Planiago alpina, Erythroniam dens-canis...

- La gispetière, pelouse discontinue de Festaca eskia, Androsace carnea. Gentiana kochiana. Cardamine resedifolia, Lazula pediformis, Silene rupestris. Trifoliam alpinum.

Il faut ajouter à ces groupements najeurs la flore fongique, qui joue un rôle trophique considérable (ectomycorhizes fréquemment dues à Boletus div. sp.), ou bien qui constitue un agent de dépérissement (Herpotrichia coutleri sur jeunes pins, Armillaria obscura sur arbres âgés, etc.).

Au-dessus des niveaux altimontains et subalpins, Pinus uncinata se rencontre occasionnellement à l'état isolé parmi les chaméphytes à la base de l'alpin sur granite, où il forme de minuscules enclaves arborées sur les croupes précocement déneigées. Le record altitudinal absolu de l'espèce est atteint au massif des Encantats, sur le territoire espagnol, avec une cote excédant $2700 \mathrm{~m}$ !

\subsection{Le substrat}

Qu'il pousse en milieu calcaire comme en Anie, ou en milicu granitique comme en Néouvielle, le pin à crochets colonise préférentiellement les croupes rocheuses. Aussi peut-on se demander si le pin est sensible à la nature chimique de la rochemère sur laquelle il pousse. Dans l'affirmative il y aurait sûrement différenciation d'écotypes édaphiques, les uns calcicoles et les autres silicicoles. En réalité cette hypothèse est à rejeter d'emblée puisque les observations de terrain et les analyses pédologiques montrent une nette analogie des sols où s'enracine Pinus uncinata, que le substrat soit granitique ou bien calcaire. Au massif d'Anie en particulier, le pin 


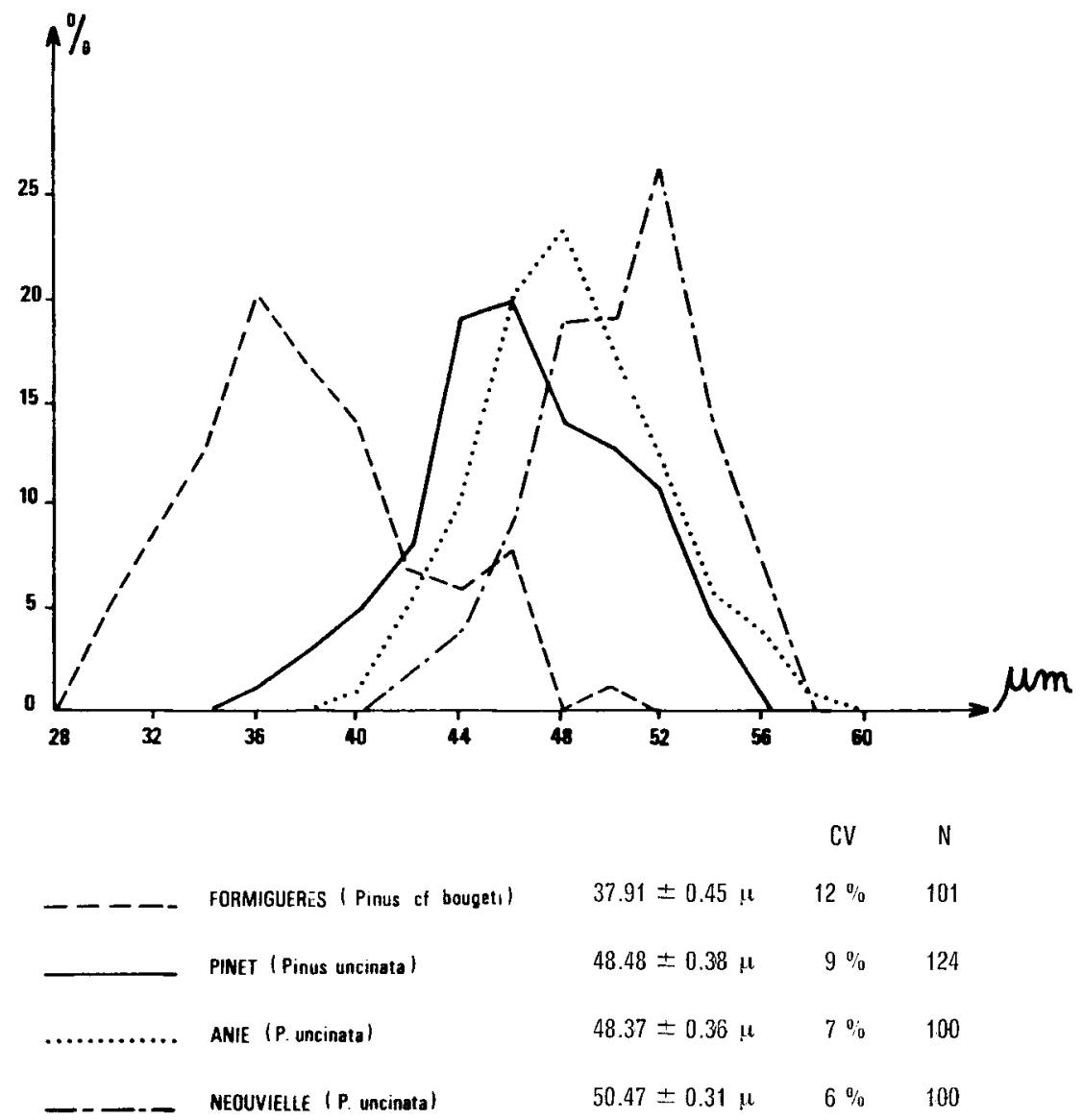

FIG. 3

Distribution diamétrale du pollen de quatre populations pyrénéennes.

Pollen diametral distribution of four Pyrenean populations.

N.B. : Conventionnellement :

- la moyenne $m$ est encadrée par son écart-type $\pm \frac{\mathrm{s}}{\sqrt{ } \mathrm{N}}$ ( $\mathrm{s}=$ écart-type des observations et $\mathrm{N}=$ effectif de l'échantillon) qui représente l'intervalle de confiance à la probabilité de 68 p. 100 ;

- la dispersion des observations autour de la moyenne $m$ est exprimée par le coefficient de variation relative $\mathrm{CV}=\frac{\mathrm{s}}{\mathrm{m}}$.

- the mean $m$ stands between its standard-deviation $\pm \frac{s}{\sqrt{ } N}(s=$ standard-deviation of measurements, $N=$ size of sample) representing confidence range with $68 \mathrm{p} .100$ probability:

- the dispersion of measurements round about the mean $m$ is expressed by relative variation coefficient $C V=\frac{s}{m}$. 
à crochets génère son propre "sol» par l'amoncellement des aiguilles sous son couvert et s'affranchit entièrement de la roche carbonatée sous-jacente : la matière organique évolue lentement vers un xéromor acide très noir reposant directement sur la dalle calcaire (CABIDOCHE, 1979).

\subsection{Le vieillissement des pineraies occidentales}

La longévité du pin à crochets est remarquable dans les Pyrénées occidentales où les arbres chenus de 500 ans ne sont pas rares. La structure démographique de ces peuplements paraît déséquilibrée par déficit des jeunes classes d'âge, mais aucun facteur prépondérant du milieu (climat, páturage, attaques parasitaires et prédatrices...) n'explique à lui seul la tendance contemporaine à la baisse du potentiel régénératif.

\section{La discrimination morphologique par les caractéristiques d'éléments végétatifs et génératifs}

Aux différences dans l'architecture des morphotypes d'Anic et de Néouvielle, la caractéristique majeure du pin d'Anie étant sa fréquente multicaulie (près du tiers de l'effectif différencie deux ou trois tiges dès la souche de l'arbre), se superposent certaines discontinuités affectant la variation cles caractères quantitatifs :

\subsection{Les pollens (fig. 3)}

La taille du corps du grain de pollen, mesurée à l'oculaire micrométrique, diffère très nettement (au risque de 1 p. 1000) suivant les populations parentales. Une anomalie apparaît dans la moyenne obtenue pour Formiguères : sa faible valeur, ainsi que la plurimodalité et l'amplitude de la distribution, font attribuer à Pinus $\times$ bougeti Flous (hybride pyrénéen $P$. sivestris $\times P$. uncinata) les arbres-pères échantillonnés en Capcir (Aytug, 1962).

\subsection{Les cônes (fig. 4,5 et 6)}

Quatre attributs du cône se révèlent très discriminants au niveau des Pyrénées pour caractériser les pineraies de Pinus uncinata : longueur et diamètre, forme de l'écusson et, à un degré moindre, excentration du pédoncule.

Les critères qualitatifs interviennent également dans la distinction des principaux massifs centraux et occidentaux, et en définitive :

- Anie et Néouvielle possèdent les mêmes types de cône, mais couleur et dimensions diffèrent ( $625,78 \mathrm{~mm}$ et $28,41 \mathrm{~mm}$ ).

- Encantats se détache par les crochets particulièrement développés, les teintes brillantes et la grosseur de ses cônes (D) 29,39 mm).

- Pinet, enfin, s'individualise grâcc à la taille réduite de ses cônes (6) $25.03 \mathrm{~mm}$ ) et aux écussons peu saillants (abondance du type rotundata).

En outre, la comparaison des tests de Duncan (fig. 6) révèle une variabilité des attributs du cône bien supérieure en Néouvielle par rapport à Anie. 


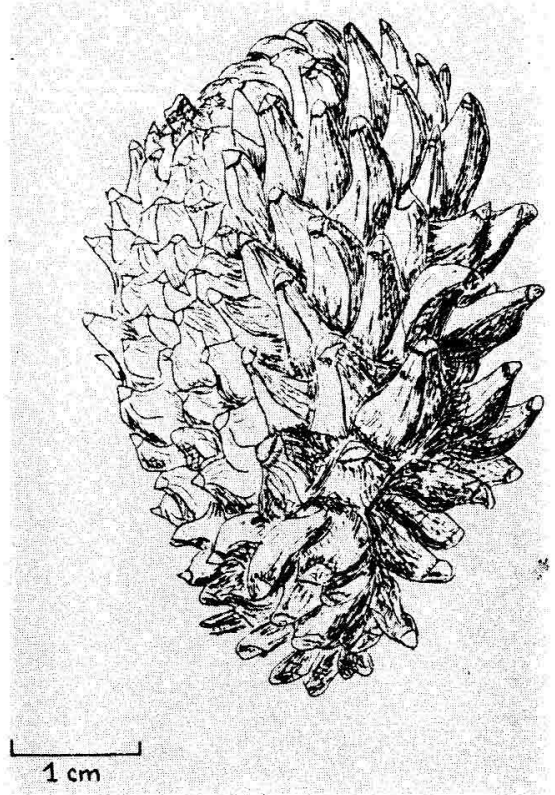

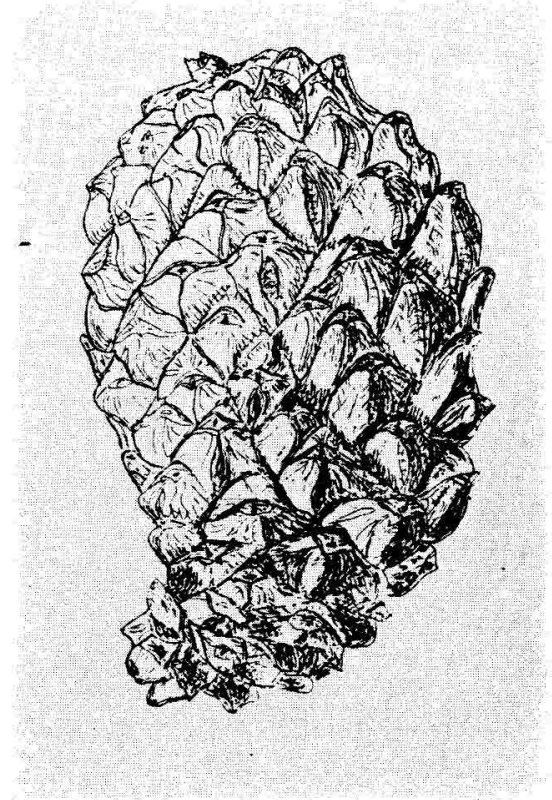

FIG. 4

Aspects des cônes chez Pinus uncinata au massif des Encantats : types rostrata et rotundata. Cone aspect of Pinus uncinata within Encantats stand : rostrata and rotundata types.

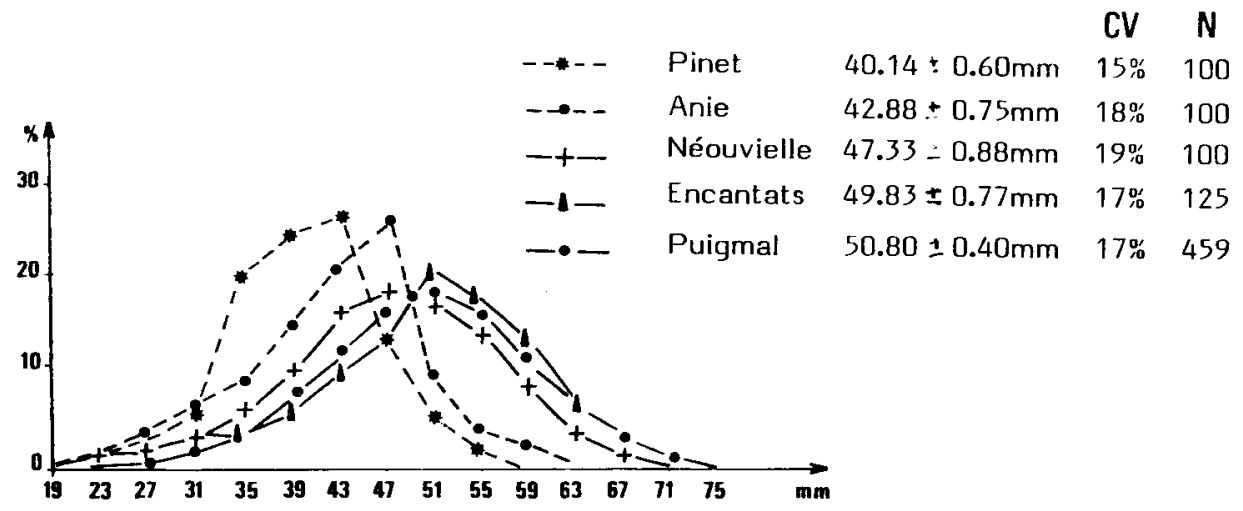

FIG. 5

Distribution de la longueur du cône dans cinq massifs pyrénéens. Length distribution of cones within five Pyrenean provenances. 

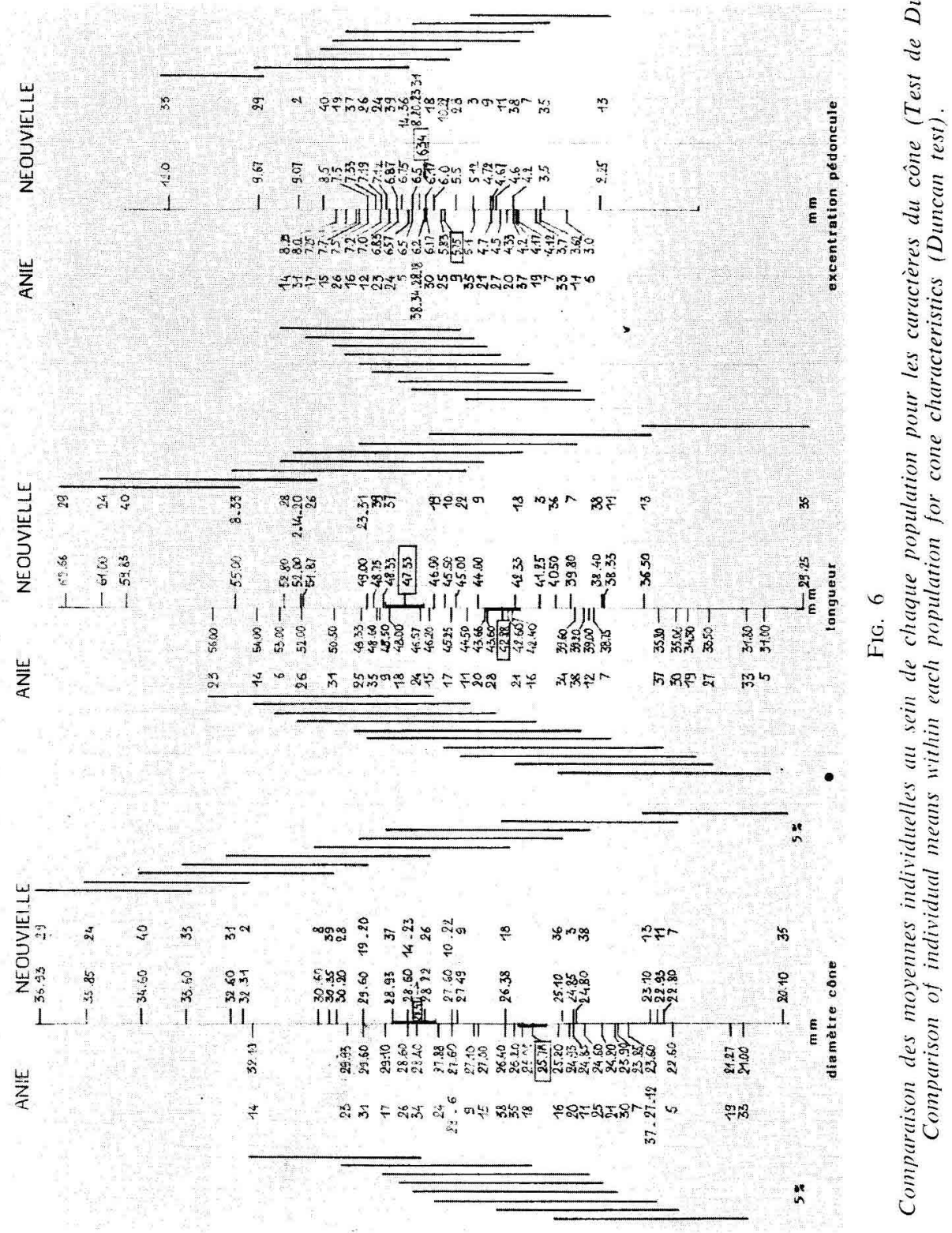


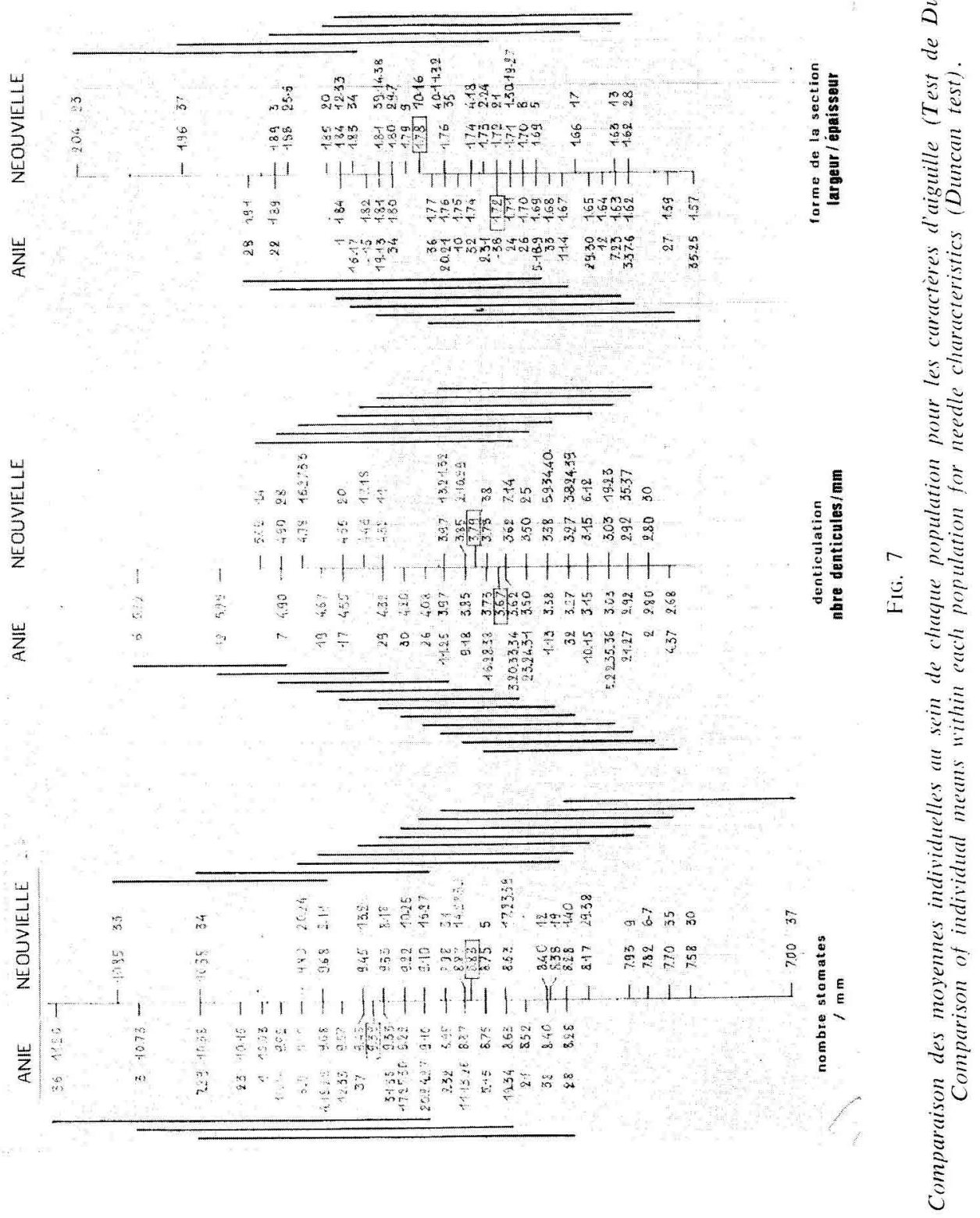


A l'issue de l'étude quantitative des organes reproducteurs du pin à crochets, on constate quau niveau provenance la variation de la taille des pollens s'effectue dans le même sens que celle des cônes :

$$
\text { Pinet }<\text { Anic }<\text { Néouvielle }
$$

Ainsi dans les Pyrénées les dimensions réduites du cône et du grain de pollen semblent caractéristiques de populations marginales, comme celles d'Anic et de Pinet.

\subsection{Les aiguilles (fig. 7)}

Les moyennes diffèrent très significativement (au risque de 1 p. 1000) pour deux caractères :

- les rangs stomatiques sont plus serrés en Anie qu'en Néouvielle : 9.39 contre 8,82 stomates par millimètre;

- la forme de la section se rapproche plus du demi-cercle en Néouvielle (rapport largeur/épaisseur $=1,78)$ qu’en Anie $(1,72)$ : les aiguilles de la pineraic karsique présentent une robustesse, donc une rigidité, plus accusées.

Les tests de Duncan (fig. 7) révèlent encore une variabilité plus importante de ces caractères en Néouvielle. En revanche le phénomène inverse se produit pour la denticulation (nombre de denticules sur les arêtes de l'aiguille) qui paraît varier indépendamment des autres critères.

\subsection{Les graines}

Après élimination des graines vaines par tri densitaire à l'éther de pétrole, les caractéristiques séminales et les tests de germination réalisés sur substrat de vermiculite humide séparent nettement les provenances, surtout Anie el Néouvielle (tabl. 2).

\section{TABI.IAU 2}

Caractéristiques des graines de Pinus uncinata dans trois massifs pyrénéens.

Seed characteristics of Pinus uncinata between three Pyrenean provenances.

\begin{tabular}{|c|c|c|c|}
\hline Caractéristique & Anie & Néouvielle & Encantats \\
\hline Proportion de gratines pleines & $57 \%$ & $73 \%$ & $79 \%$ \\
\hline Masse de 1000 graines & $9.07 \pm 0.06 \mathrm{~g}$ & $8.41 \pm 0.05 \mathrm{~g}$ & $8.38 \pm 0.01 \mathrm{~g}$ \\
\hline Nombre de graines/kg & 110300 & 118900 & 119300 \\
\hline $\begin{array}{l}\text { Faculté germinative (germes vivants ì } \\
6 \text { semaines) } \ldots \ldots \ldots \ldots \ldots \ldots \ldots \ldots\end{array}$ & $75 \%$ & $59 \%$ & $76 \%$ \\
\hline
\end{tabular}

Pour assurer leur régénération, les deux populations occidentales adoptent des stratégies inverses : 
- Anie libère peu de graines pleines, mais elles se caractérisent par lcur masse importante et une bonne faculté germinative :

- Néouvielle en revanche produit beaucoup de graines, mais plus légères et à germination défectueuse.

Les performances régénératives des Encantats, d'après les essais en germoir effectués en 1980, se révèlent bien supérieures.

\subsection{Les plantules (fig. 8 et 9)}

Après la germination épigée de la graine, se développent les colylédons dont le nombre est très variable et non spécifique chez les Gymnospermes. Cependant le dénombrement des cotylédons s'avère parfois fort utile pour la séparation de groupes géographiques. Ici, chez Pinus uncinata, la comparaison en pépinière des descendances de trois populations montre que le nombre moyen de cotylédons diffère très significativement entre Anie $(5,71)$ et Néouvielle $(5,10)$ et beaucoup moins nettement entre Néouvielle et Encantats $(5,27)$.
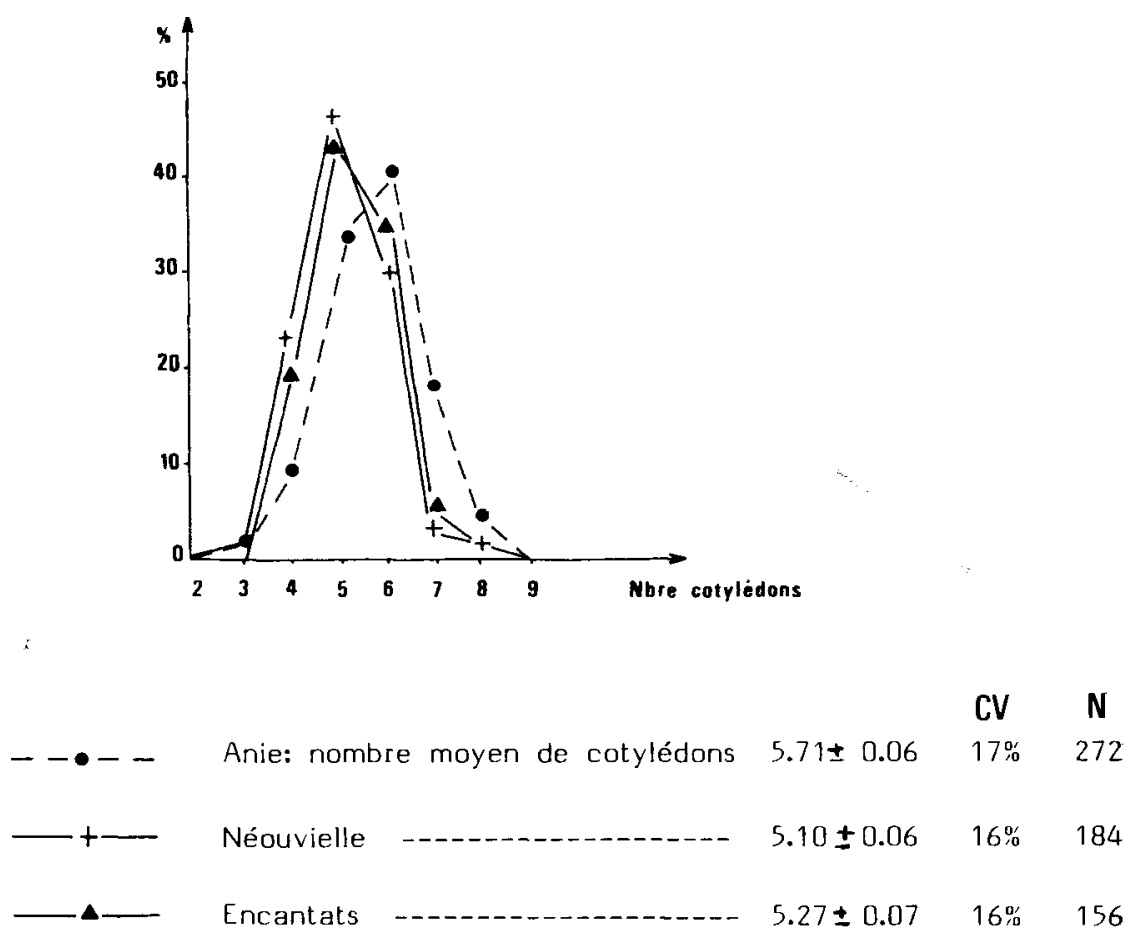

Fig. 8

Profil cotylédonaire des descendances de trois pomulutions pyrinesonnes.

Cotyledonian outline of outsprings of three pyrenean populations. 


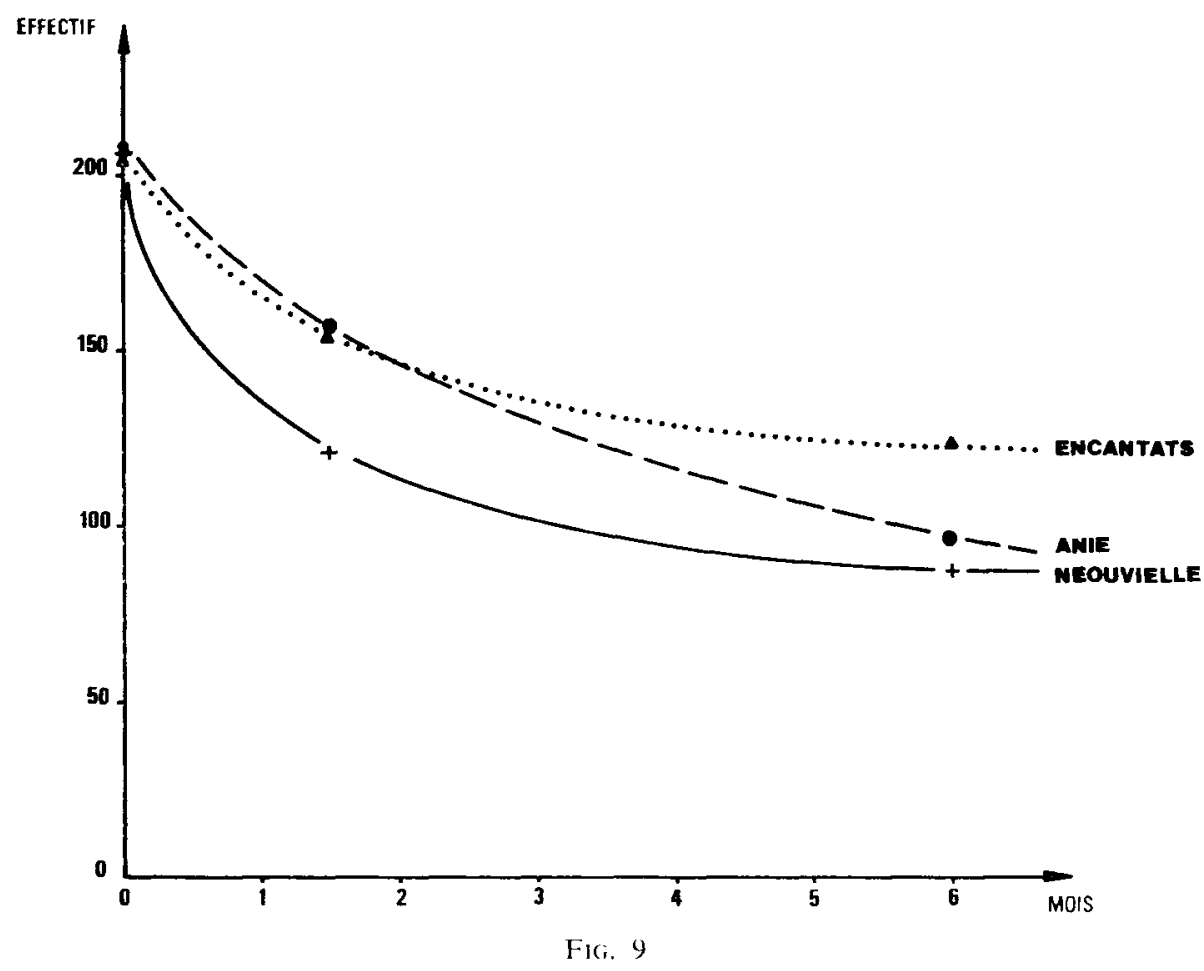

Survie des plantules de Pinus uncinatal pendant la première saison de végétation an pépinière. Seedling outliving of Pinus uncinata during first vegetative season in mursery.

Si l'on se réfère à la masse séminale, on observe que la provenance d'Anie, avec des graines lourdes, donne naissance à des plantules bien pourvues en cotylédons. Au bout de six mois en pépinière, les semis des populations occidentales se retrouvent à peu près au mème niveau numérique, avec une importante mortalité post-germinative (38 p. 100) des descendances d'Anie, alors qu'aux Encantats les pertes n'atteignent que 21 p. 100 des effectifs à six semaines.

Il semble que l'on puisse d'abord attribuer la bonne survie des plantules issues des Encantats à une meilleure résistance à la fonte des semis.

*

Jusqu’ici, cette étude biosystématique se borne à comparer des attributs morphologiques provenant de populations naturelles écologiquement très diversifiées. Ainsi, malgré des différences biostatistiques non négligeables, les massifs d'Anie et de Néouvielle ne se distinguent-ils que par des critères phénotypiques pour lesquals on ne peut séparer l'effet génotypique de l'effet environnemental. A ce stade, on n'apporte donc pas la preuve formelle d'une discontinuité génétique entre les deux populations. 


\section{L'utilisation des hydrocarbures terpéniques comme marqueurs génétiques}

La supériorité taxonomique des analyses biochimiques réside dans le fait que certains composés (enzymes, terpènes, ...) possèdent une variation discontinue, plurimodale, à l'intérieur d'une provenance (fig. 10), révélant un contrôle génétique simple, contrairement aux caractères métriques où la distribution prend l'allure d'une courbe en cloche, indice d'un déterminisme multifactoriel (polygènes, interactions avec le milieu). On a alors réalisé pour la première fois chez Pinus uncinata l'analyse de marqueurs biochimiques en vue d'une interprétation génétique. A cet effet, l'analyse des composants de l'oléorésine des tissus corticaux, et spécialement de la fraction mono- et sesquiterpénique séparée par chromatographie en phase gazeuse (fig. 11), confirme que plusieurs hydrocarbures sont vraisemblablement conditionnés par un faible nombre de gènes et aboutit aux résultats suivants :

- il n'existe aucune différence qualitative des essences corticales cntre Anie et Néouvielle : la répartition des individus en six types monoterpéniques est à peu près la même dans chacune des deux populations. On note par ailleurs la quasiabsence du delta-3-carène chez ces deux provenances de Pinus uncinata, et il serait intéressant, s’il s'agit là d'une règle généralisable à l'aire pyrćnćcnne, de tester la valeur de ce composé pour séparer $P$. uncinata et $P$. sivestris, et éventuellement pour caractériser les hybrides interspécifiques;

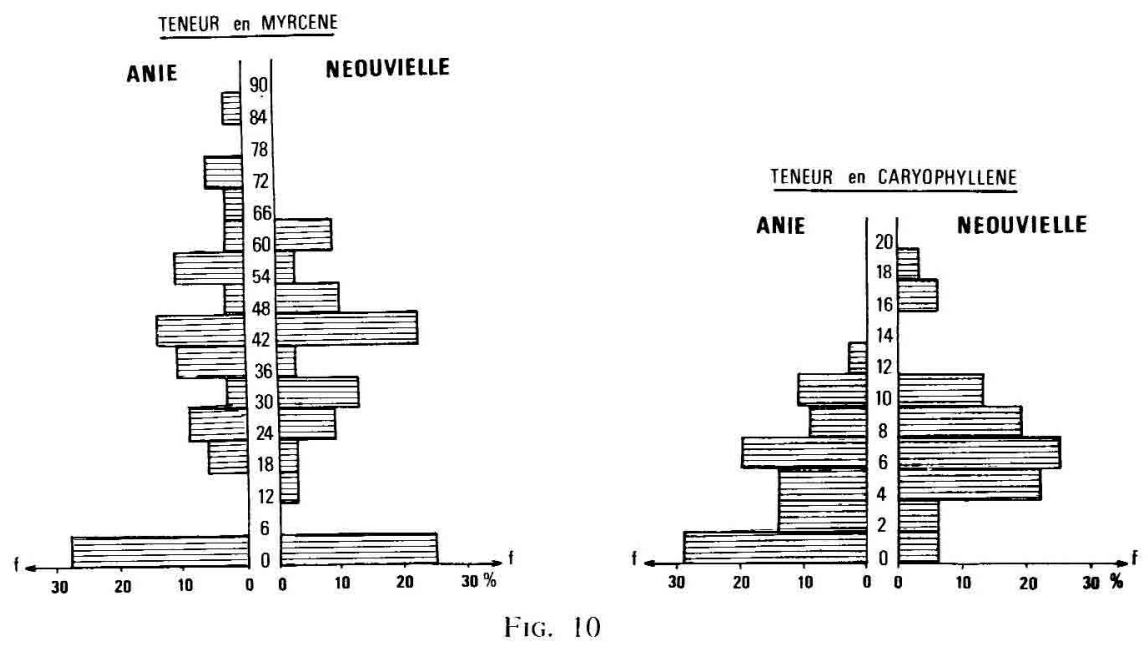

Analyse terpénique des tissus corticaux de Pinus uncinata:

variation des teneurs au sein de chaque population pour deux composés majeurs.

Terpenic analysis of cortical tissues of Pinus uncinata :

the variation of concentration within every population for two important components.

N.B. :

- la teneur est exprimée en pourcentage du total mono- + sesquiterpènes;

- $\mathrm{f}$ représente la fréquence relative des individus (arbres).

- the concentration is expressed by the total mono- + sesquiterpenes percentage;

- $f$ shows the relative frequency of individuals (trees). 


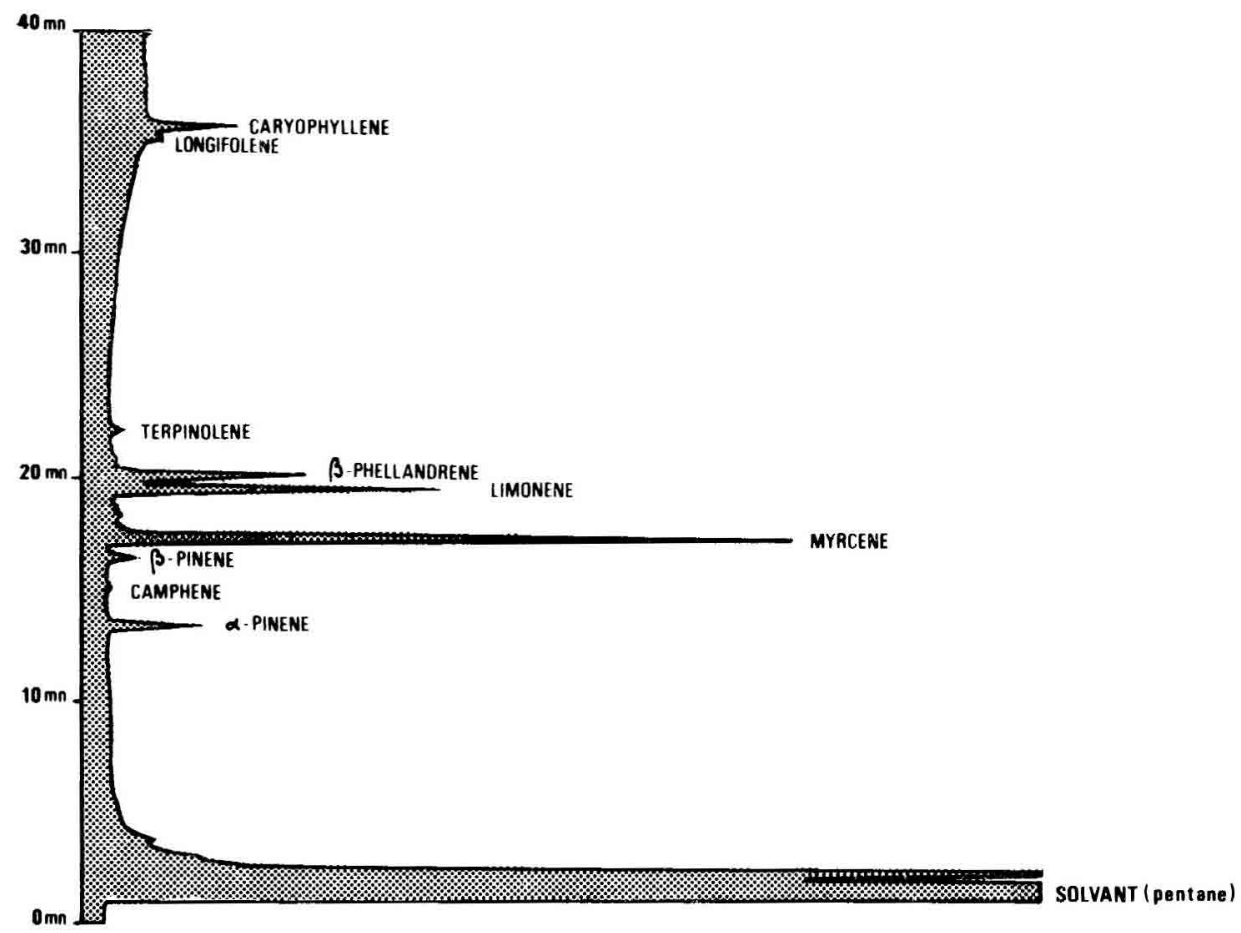

FIG. 11

Exemple de chromatogramme d'analyse terpénique chez. Pinus uncinata.

Terpenic analysis of cortical tissues of Pinus uncinata :

N.B. : L'échelle minutée représente les temps de rétention des différents monoterpènes et sesquiterpènes.

Scale in minutes shows the retention times of different monoterpenes and sesquiterpenes.

- on observe cependant des phénotypes sensiblement plus riches en Néouvielle qu'en Anic, notamment les teneurs en pinènes, en bêta-phellandrène et en caryophyllène (fig. 12).

Ces différences quantitatives mises en évidence militent en faveur d'une individualisation d'Anie par rapport à Néouvielle, non plus simplement morphologique, mais probablement aussi génćtique.

A l'issue de l'étude séparée des caractères, on peut admettre en conclusion l'existence aux Pyrénées occidentales de deux taxons distincts de pin à crochets dont le plus interne, Néouvielle, accuse une large variabilité morphologique et terpénique. Néanmoins, l'analyse des marqueurs biochimiques semble indiquer une différenciation génétique moins évidente que celle pressentie lors de l'étude biométrique. 


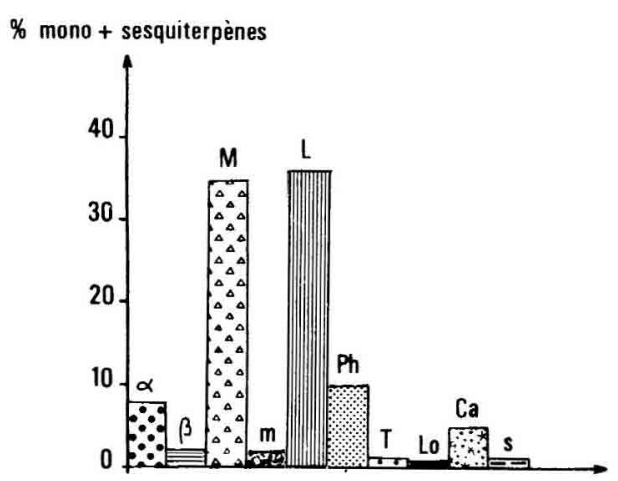

A N I E

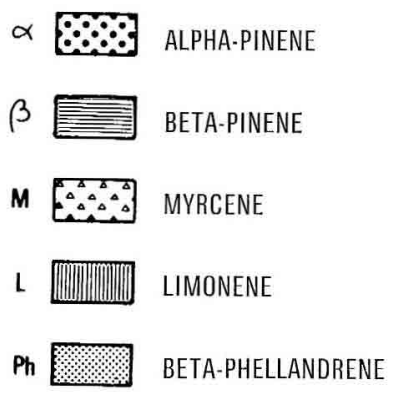

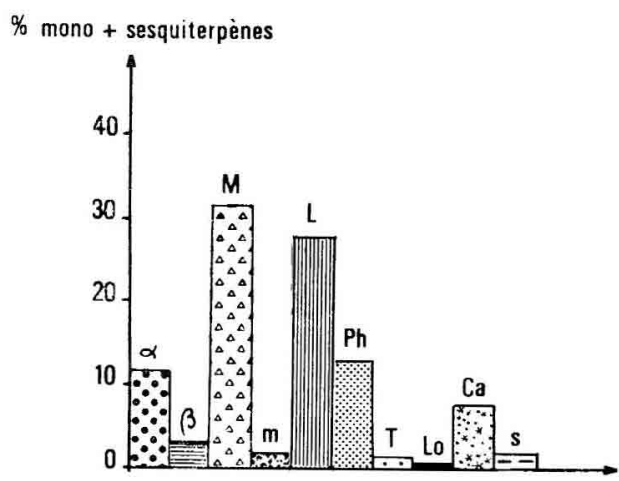

NE OUVIELLE

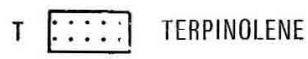

m

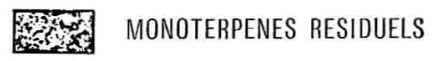

Lo

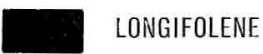

Ca CARYOPHYLLENE

$s$ SESQUITERPENES RESIDUELS

FIG. 12

Profils terpéniques des tissus corticaux chez deux populations de Pinus uncinata : teneurs moyennes en mono- et sesquiterpènes.

Terpenic patterns of cortical tissues within two populations of Pinus uncinata : mono- and sesquiterpenes average concentrations.

\section{Déformations dues au milieu et liaisons entre caractères}

\subsection{Effets de l'environnemeni}

Sans insister ici sur les déformations subies par le pin au niveau de son architecture externe, on désire à présent tester l'effet environnemental sur la variation des caractères biochimiques et morphologiques mesurés au sein de chaque population. A cette fin, la construction de matrices de corrélations entre cáractères métriques et variables du milieu (topographie, pente, exposition, fragmentation du substrat, structure du peuplement) montre une influence négligeable des caractéristiques stationnelles sur les organes en cause, excepté sur la longueur de l'aiguille et sur la masse des graines, directement sensibles aux conditions trophiques de l'arbre. En revanche l'altitude possède l'incidence la plus marquée : 


\subsection{Effet de l'altitude sur la morphologie des aiguilles}

- la longueur diminue avec l'altitude en Néouvielle $\left(r=-0.722^{* *}\right)$ (1). En Anie, la variation est discontinue, les aiguilles les plus courtes se trouvant aux altitudes extrêmes;

- la persistance est également liée négativenent à l'altitude en Anie $\left(\mathrm{r}=-0,577^{* * *}\right)$;

- le nombre de lignes stomatiques croît vers les sommets en Néouvielle $(r=0,429 * *)$.

\subsection{Effet de l'altitude sur la régénération}

- la faculté germinative décroît plus neltement en Anie $\left(r=-0,561^{* *}\right)$ quen Néouvielle $\left(\mathrm{r}=-0,385^{*}\right)$;

- Le nombre de cotylédons des plantules augmente en Néouvielle $\left(r=0,527^{*}\right)$.

\subsection{Effet de l'altitude sur la composition terpénique}

Uniquement en Néouvielle, on détecte une variation peu sensible de certaines teneurs en terpènes (coefficiont de corrélation de rang dî̀ à Spearman significatif au risque de 10 p. 100) :

-- bêta-phcllandrène : $r_{s}=-0,318$;

- caryophyllène $\quad: r_{s}=0,328$.

En définitive, seul le cône semble échapper à l'emprise du milieu cxtérieur, ce qui confirme la valeur de cet organe dont les dimensions et l'ornementation sont traditionnellement prises en compte dans les études biosystématiques. D’autres éléments, au contraire, subissent pour certains de leurs caractères métriques des biais manifestes dus à l'environnement, tels que pseudophylles. graines et plantules.

Les essences corticales du pin vérifient une bonne écostabilité avec cependant en Néouvielle une très légère variation de teneur en altitude pour deux hydrocarbures. Ne peuton voir là, dans ce massif granitique à fort dénivelé, l'indice d'une différenciation génétique des peuplements d'altitude?

\subsection{Effets de la vigueur et de l'âge}

A l'impact des conditions environnantes s'ajoute une autre source de variation potenticlle sur les caractères mesurés, qui s'exprime par l’âge et la vigueur de l'arbre sur lequel sont prélevés les divers éléments étudiés. En réalité les critères dendrométriques pris isolément (grosseur de l'arbre. vicillissement, etc.) n’interviennent que de façon négligcable, aussi l'estination des valeurs de chaque caractère incriminé (variable expliquéc) se fait-elle par un ensemble de 6 prédicteurs grâce à une analyse de régression multiple au sein de chaque population (tabl. 3).

(1) Signification des coefficients de corrélation linéaire dans ce mćnoìe :

$\mathrm{f}^{*}$ : significatif au risque de 1 p. 100.

$r^{*}:$ significatif au risque de $5 \mathrm{p}, 100$,

$r \quad$ : significatif au risque de 10 p. 100 ,

NS : non significatif. 
TABleau 3

Corrélations multiples entre caractères biochimiques ou morphologiques et critères de vigueur du pin à crochets.

Multiple correlations between biochemical or morphological characteristics and vigor data of mountain pine.

\begin{tabular}{|c|c|c|c|c|c|}
\hline \multirow{2}{*}{ Groupe } & \multirow{2}{*}{ Variable expliquée } & \multicolumn{2}{|c|}{ Anie } & \multicolumn{2}{|c|}{ Néouvielle } \\
\hline & & coefficient & F 6 d.l. & coefficient & F 6 d.I. \\
\hline & alpha-pinène & - & - & $0,375^{*}$ & $<1$ \\
\hline & iimonène ... & $0,576 * *$ & 2.32 & - & - \\
\hline & bêta-phellandrène ... & $0,5,56 * *$ & 2,09 & $0,379 *$ & $<1$ \\
\hline Terpènes & monoterpènes résiduels & - & - & $0,540^{* * *}$ & $1,72 \mathrm{NS}$ \\
\hline A : 28 d.l. & longifolène $\ldots \ldots \ldots$ & $0,693 * *$ & $4,30 * *$ & - & - \\
\hline $\mathrm{N}: 25$ d.I. & caryophyllène ..... & - & 一 & $0,543 * *$ & $1,74 \mathrm{NS}$ \\
\hline Aiguille & épaisscur & $0,481 * *$ & $1,46 \mathrm{NS}$ & $0,516^{* *}$ & $1.69 \mathrm{NS}$ \\
\hline A : 29 d.1. & largeur $\ldots$. & $0,679 * *$ & $4,1,5^{* * *}$ & - & - \\
\hline $\mathrm{N}: 28$ d.I. & denticulation & - & - & $0,363 *$ & $<1$ \\
\hline $\begin{array}{l}\text { Graine } \\
\mathrm{A}: 21 \text { d.l. }\end{array}$ & faculté germinative & $0,567 * *$ & $1,66 \mathrm{NS}$ & - & $一$ \\
\hline Cône & longueur & $0,601^{* *}$ & $1.88 \mathrm{NS}$ & - & - \\
\hline A : 20 d.l. & largeur écusson & $0,729: * *$ & $3.79^{*}$ & 一 & - \\
\hline
\end{tabular}

N.B. : A : Anie; N : Néouvielle; F : test de l'apport global des six variables explicatives dans la variance totale du caractère - test of global contribution of the six explanatory variables in the total variance of characteristics.

On remarque que certains caractères terpéniques peuvent dépendre de l'état végétatif de l'arbre, d'où l'intérêt d'opérer les ajustements décrits ci-dessus pour établir des comparaisons rigoureuses entre Anie el Néouvielle.

La confrontation des caractéristiques du pin aux variables écologiques et dendrométriques majeures permet de détecter certains critères directement sensibles aux sollicitations du milicu extérieur ou aux elfets de vigueur, qui en conséquence n'émettent qu'un «bruit de fond» dans la recherche de discontinuités génétiques entre peuplements de pin à crochets.

Mais d'autre part, on peut se demander s'il n'existe pas d'interférences entre les divers critères mesurés, si des redondances ne viennent pas alourdir exagérément l'information prélevée dans les populations naturelles.

\subsection{Liaison entre caractères}

\subsection{Corrélations phénolypiques}

- entre caractères de l'aiguille (fig. 13)

Malgré des conditions environnantes spéciales à chaque massif, les graphes d'Anie et de Néouvielle s'avèrent nettement apparentés, ce qui tend à prouver que le schéma relationnel pour les caractères des aiguilles possède une stabilité satisfaisante : 


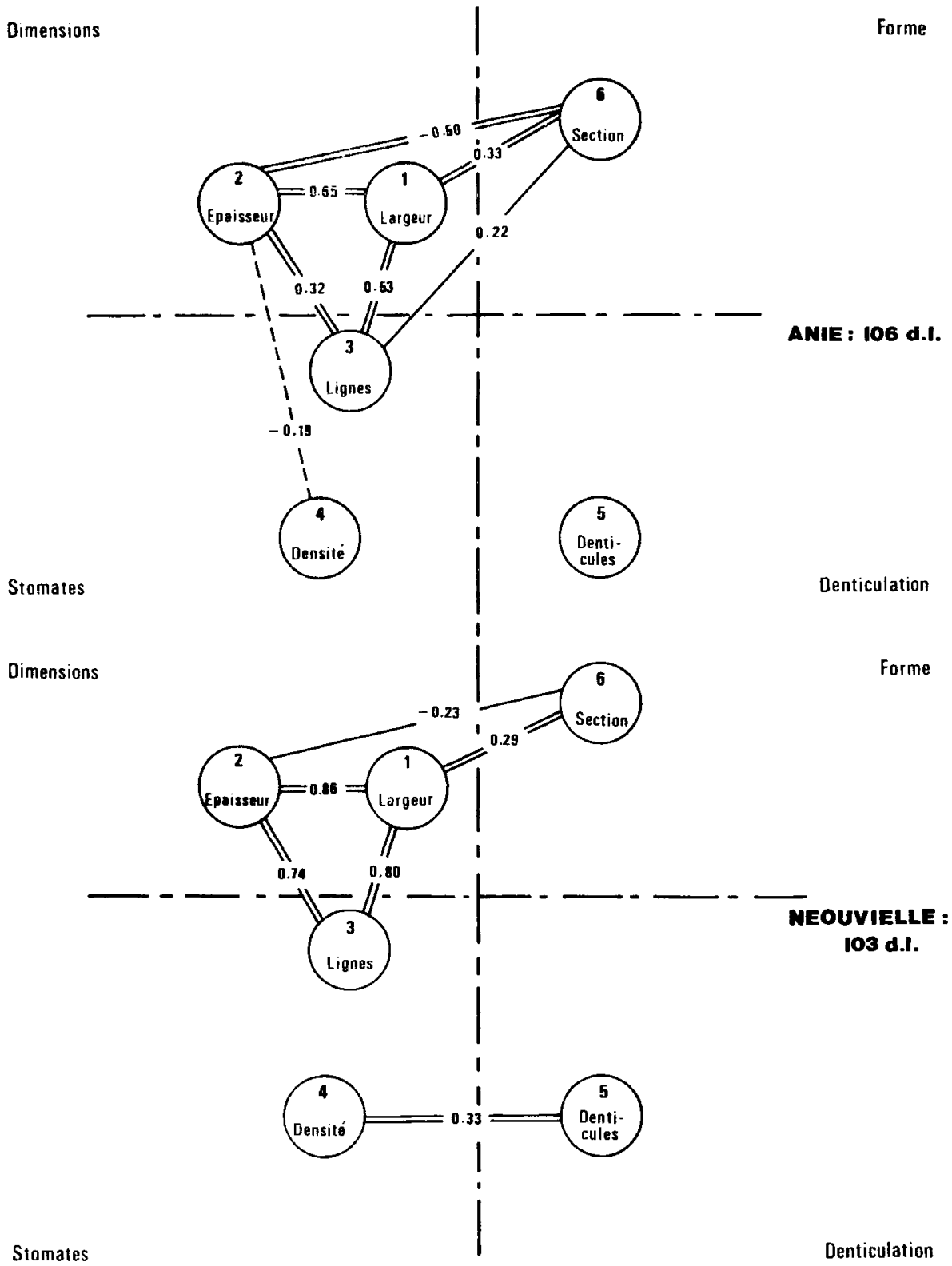

FIG. 13

Graphe des corrélations phénotypiques entre caractères d'aiguille au sein des populations Anie et Néouvielle.

Graph of phenotypic correlations between needle characteristics within Anie and Neouvielle populations.

N.B. : Signification des corrélations - Correlation signification :

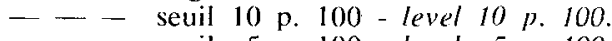

seuil 5 p. 100 - level 5 p. 100.

seuil 1 p. 100 -level 1 p. 100. 


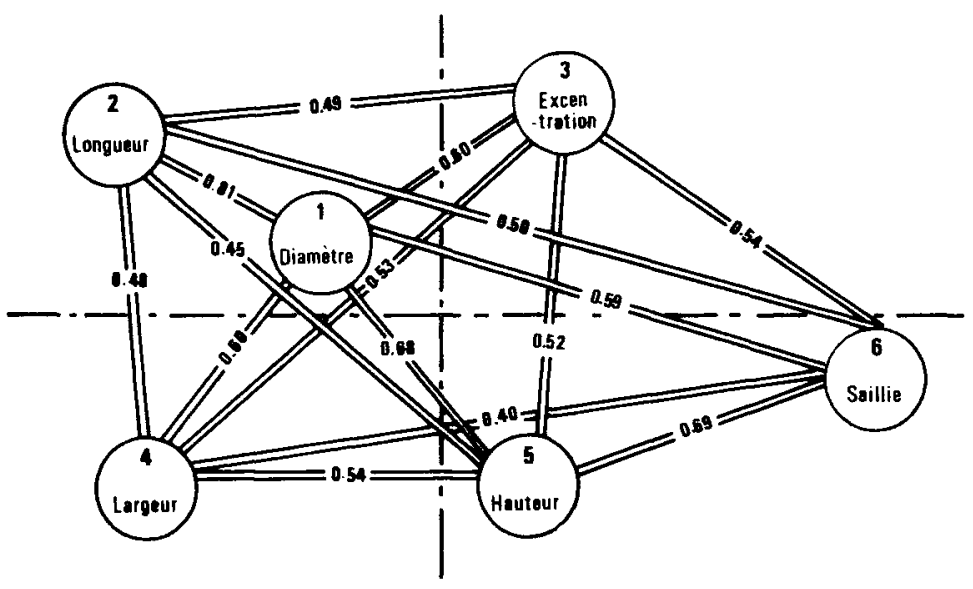

Ecaille

Crochet

ANE: 98 d.I.

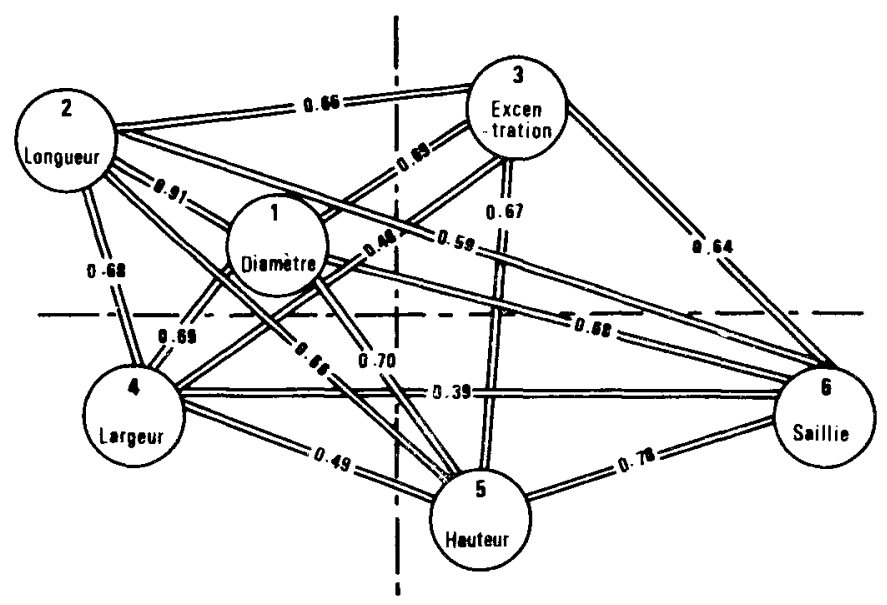

Ecaille

Crochat

MEOUNELAE : 98 d.l.

Fig. 14

Graphe des corrélations phénotypiques entre caractères du cône au sein des populations Anie et Néouvielle.

Graph of phenotypic correlations between cone characteristics within Anie and Neouvielle populations. 
- forte liaison des critères dimensionnels;

- corrélation positive entre dimensions transversales de l'aiguille et nombre de lignes stomatiques;

— indépendance de ces critères avec les densités de stomates et de denticules.

- entre caractères du cône (fig. 14)

Tous les caractères se révèlent fortement liés entrc-eux, et de façon positive : les cônes les plus gros sont les plus asymétriques et présentent aussi les crochets les micux développés.

\subsection{Similarité des caractères}

A partir de 24 critères morphologiques et biochimiques dont certains, plus affectés par l'âge ou la vigueur de l'arbre, sont préalablement ajustés, l'étude des similarités consiste à établir une matrice de corrélations puis à constituer des groupes de caractères par niveau de similarité décroissant. Les coefficients de similarité ne sont autres que les valeurs absolues des corrélations linéaires si bien que plus le seuil de similarité est faible et plus les caractères s'assemblent pour ne former qu'un seul groupe au niveau proche de zéro.

La visualisation de l'affinité entre caractères se présente sous forme d'un schéma ramifié ou dendrogramme dont on donne ici un exemple établi pour la population d'Anie (fig. 15).

NIVEAU DE SIMILARITE DÉCROISSANT

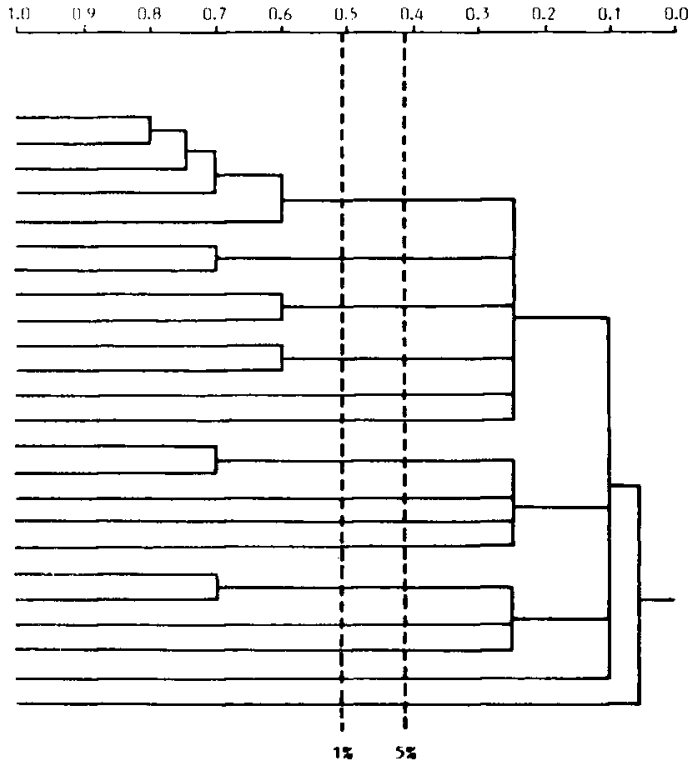

ANII: 22 d.I

$\left\{\begin{array}{l}\text { diamètre } \\ \text { hauteur écusson } \\ \text { excentration du pédoncule } \\ \text { sáillie de l'écusson } \\ \text { longueur } \\ \text { sesquilerpènes résiduels } \\ \text { terpinolène } \\ \text { alpha-pinène } \\ \text { bêta-pinène } \\ \text { caryophyllène } \\ \text { monoterpènes résiduels } \\ \text { longifolène } \\ \text { bêta-phellandrène } \\ \text { limonène } \\ \text { mayrcène } \\ \text { denticulation } \\ \text { ponctuation linéique } \\ \text { faculté gorminative } \\ \text { largeur } \\ \text { épaisseur } \\ \text { linéation stomatique } \\ \text { nombre de cotylédons } \\ \text { largeur de l'écusson } \\ \text { masse des graines }\end{array}\right.$

masse des graines

Fig. 15

Dendrogramme-Anie : liaison entre caractères morphologiques et biochimiques. Anie dendrogramm : connexion between morphological and biochemical characteristics. 
Les caractères manifestement les plus liés entre-eux sont ceux du cône, ce qui confirme les résultats obtenus précédemment et milite en faveur d'une détermination polygénique commune des différents attributs de cet organe. De même, les dimensions transversales de l'aiguille se trouvent-elles nettement corrélées et pratiquement indépendantes de la densité linéaire des stomates et des denticules.

Les composés terpéniques forment un bloc relativement isolé des attributs morphologiques et jouissent certainement d'un déterminisme original. L'existence de quelques couples de terpènes suggère l'intervention de mécanismes génétiques tels que linkage ou pléiotropie, ainsi les teneurs en myrcène et limonène apparaissent-elles fortement interdépendantes au sein de chaque population. On sait par ailleurs que les gènes qui contrôlent ces deux terpènes sont effectivement étroitement liés chez le pin maritime, avec un taux de recombinaison de 0,12 (Ph. BARADAT, communication personnelle).

L'élimination d'une part des caractères sensibles au milieu, d'autre part des caractères redondants, permet un gain de temps et une économie de moyens appréciables pour des recherches ultérieures sur la variabilité du pin à crochets.

\section{L'éloignement taxonomique évalué par l'analyse multivariable}

En réalité il peut sembler artificiel de chercher à discriminer des groupes géographiques par une juxtaposition de variables dont la matrice de corrélations révèle la redondance. Ainsi, l'étude séparée des caractères fournit-elle une liste de variables qui discriminent Anie et Néouvielle, tous ces caractères se situant a priori sur un pied d'égalité.

L'intérêt de l'analyse multivariable est d'obtenir une combinaison linéaire de plusieurs caractères intervenant à des degrés divers dans la séparation des groupes. Cette synthèse multidimensionnelle, obtenue ici par l'analyse discriminante appliquéc à certains attributs morphologiques (cônes, aiguilles) et biochimiques (terpènes), fát apparaître une très nette divergence entre les deux populations. Bien plus, la comparaison des distances généralisées ( $\mathrm{D}^{2}$ de Mahalonobis) séparanl Anie et Néouvielle montre que lindex terpénique discrimine les deux mossifs plus nettenent encore que les caractères de cône et d'aiguille.

Si l'on s’intéresse uniquement aux caractères tcrpéniques, plus fiables que les attributs morphologiques car beaucoup plus héritables, et donc moins soumis à l'effet station (BERNARD-DAGAN \& BARADAT, 1977), les fonctions discriminantes sont sensiblement concordantes que l'on applique l'analyse canonique aux deux populations (Anie et Néouvielle), ou bien à six tranches d'altitude (trois en Anie et trois en Néouvielle) :

$$
\begin{aligned}
& Y_{1}^{\prime}=0,051 X_{1}+0,292 X_{2}-0,008 X_{: 3}-0,005 X_{1}+0,0006 X_{. ;}+0,300 X_{6}- \\
& 0,231 x_{i}-0,868 x_{s}+0,084 x_{5}+0,089 x_{111} \\
& \text { (2 populations) } \\
& Y_{1}=-0,028 X_{1}-0,284 X_{2 .}+0,007 X_{3}+0,004 X_{4}+0,005 X_{i}-0,371 X_{6}+ \\
& 0,223 X_{i}+0,847 X_{x}-0,073 x_{41}-0,087 x_{11} \\
& \text { (6 classes altitudinales) }
\end{aligned}
$$

On observe simplement que les coefficients des dix caractères sur les deux axes canoniques $Y_{1}^{\prime}$ et $Y_{1}$ sont voisins en valeur absolue et de signe inverse. Ils tendent à donner plus de poids aux teneurs en caryophyllène $X_{y}$ et en limonène $X_{1}$ (tabl. 4). 


\section{Tableau 4}

Liaisons entre les deux premiers axes canoniques et les caractères terpéniques (corrélations à 4 degrés de liberté).

Connexion baween the two first canonical axis and terpenic characteristics 14 degrees of freedom correlations).

\begin{tabular}{|c|c|c|}
\hline Axe canonique & $Y_{t}$ & $Y_{3}$ \\
\hline Valeur propre..$\ldots \ldots \ldots \ldots$ & 0.732 & 0.355 \\
\hline$\because$ de discrimination $\ldots .$. & 50.60 & 24,54 \\
\hline$\%$ de discrimination cumulé & 50,60 & 75,14 \\
\hline$X$, alpha-pinène & $-0,487$ NS & $\ldots 0,151 \mathrm{NS}$ \\
\hline$X_{2}$ bêta-pinène & $-0.721 \mathrm{NS}$ & $0.323 \mathrm{NS}$ \\
\hline$X_{:}$myrcène $\ldots \ldots \ldots \ldots \ldots \ldots \ldots$ & $0,314 \mathrm{NS}$ & $\longrightarrow 0.750$ \\
\hline $\mathrm{X}$, limonène $\ldots \ldots \ldots \ldots$ & $0,828 *$ & $0,514 \mathrm{NS}$ \\
\hline$X$ : phellandrène $\ldots \ldots \ldots$ & $-0,169 \mathrm{NS}$ & $0.639 \mathrm{NS}$ \\
\hline$X_{\text {: terpinolène } \ldots \ldots \ldots \ldots \ldots \ldots \ldots \ldots \ldots \ldots \ldots \ldots}$ & -0.778 & $0.473 \mathrm{NS}$ \\
\hline$X_{i}$ monoterpènes résiduels $\ldots \ldots \ldots \ldots$ & $0.459 \mathrm{NS}$ & -0.776 \\
\hline X. longifolène $\ldots \ldots \ldots \ldots \ldots \ldots$ & $0.616 \mathrm{NS}$ & $0,704 \mathrm{NS}$ \\
\hline$X_{i}$, caryophyllène $\ldots \ldots \ldots \ldots \ldots \ldots$ & $-0,922 * *$ & $-0.102 \mathrm{NS}$ \\
\hline$X_{111}$ sesquiterpènes résiduels $\ldots \ldots \ldots \ldots$ & -0.802 & $0.361 \mathrm{NS}$ \\
\hline
\end{tabular}

La dispersion des peuplements est illustrée sur le graphique de la figure 16 où l'on a projeté leurs centres de gravité dans le plan des deux premiers axes qui expliquent les trois quarts de la discrimination. On y remarque la tendance à l'agrégation effective, selon les populations d'origine, des peuplements altitudinaux, alors même que le niveau provenance n'est plus conservé dans l'analyse. C'est dire, ct l'analogie (au signe près) des équations $\mathrm{Y}^{\prime}{ }_{1}$ et $\mathrm{Y}_{1}$ le confirme, que la discrimination obtenue sépare bien davantage des groupes géographiques (Anie et Néouvielle) que des tranches d'altitude. Par ailleurs, une distinction se fait jour dans leur mode de regroupement : les peuplements d'Anie s'alignent préférentiellement selon l'axe 2, tandis que ceux de Néouviclle se superposent le long du premier axe.

Quoi qu'il en soit, la stratification de chaque population en classes altitudinales montre qu'il n'existe pas d'effet linéaire de l'altitude sur la composition terpénique, et ne permet pas de se prononcer sur une différenciation génétique des hauts peuplements à cause d'un échantillonnage trop sommaire.

L'analyse multivariable renforce la valeur de la discrimination déjà mise en évidence par l'étude séparéc des caractères, et tend à donner plus de poids aux critères terpéniques. Elle confirme l'individualisation génétique d'Anie par rapport à Néouvielle. 


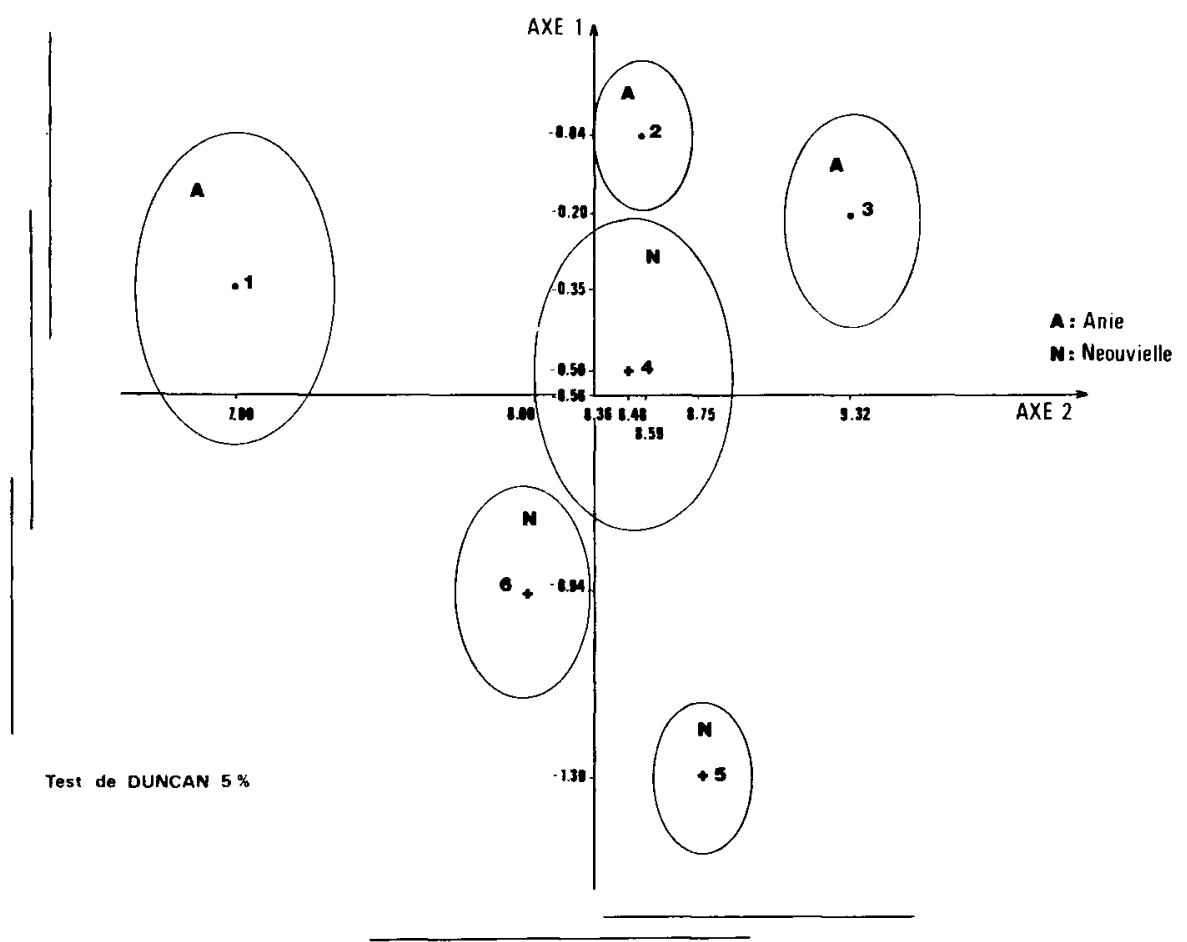

FIG. 16

Discrimination multivariable des peuplements altitudinata d'après la composition terpénique.

Multivariate discrimination of altitudinal stands based on terpenic pattern.

N.B. : Les deux premiers axes expliquent 75 p. 100 de la discrimination totale : Axe 1: richesse en limonène, pauvreté en caryophyllène et sesquiterpènes résiducls: Axe 2 : pauvreté en myrcène et monoterpènes résiduels :

- chaque centroïde cst entouré de son ellipse de confiance,

- le long de chacun des axes, les traits relient des centroïdes non significativement différents (test de Duncan au seuil de 5 p. 100).

The two first axis explain $75 \mathrm{p} .100$ of total discrimination :

Axis 1: abundance of limonene, indigence of caryophyllene and residual sesquiterpenes:

Axis 2 : indigence of myrcene and residual monoterpenes:

- each centroïd is surrounded with its confidence ellipse,

- along every axis, the lines comnect non significantly differem centroïds (Duncan test at 5 p. 100 (evel).

Classes altitudinales - Altitudinal classes :

$1=$ Anie basse altitude (1 625-1 $743 \mathrm{~m})$ - Anie low altitude.

$2=$ Anie moyenne altitude (1 743-1 $862 \mathrm{~m})$ - Anie middle altitude.

$3=$ Anie haute altitude $(1862-1980 \mathrm{~m})$ - Anie high altitude.

$4=$ Néouvielle basse altitude $(1830-2017 \mathrm{~m})-$ Neouvielle $l o w$ altitude.

$5=$ Néouvielle moyenne altitude $(2017-2203 \mathrm{~m})$ - Neouvielle middle altitude.

$6=$ Nćouvielle haute altitude $(2203-2390 \mathrm{~m})$ - Neouvielle high altitude. 


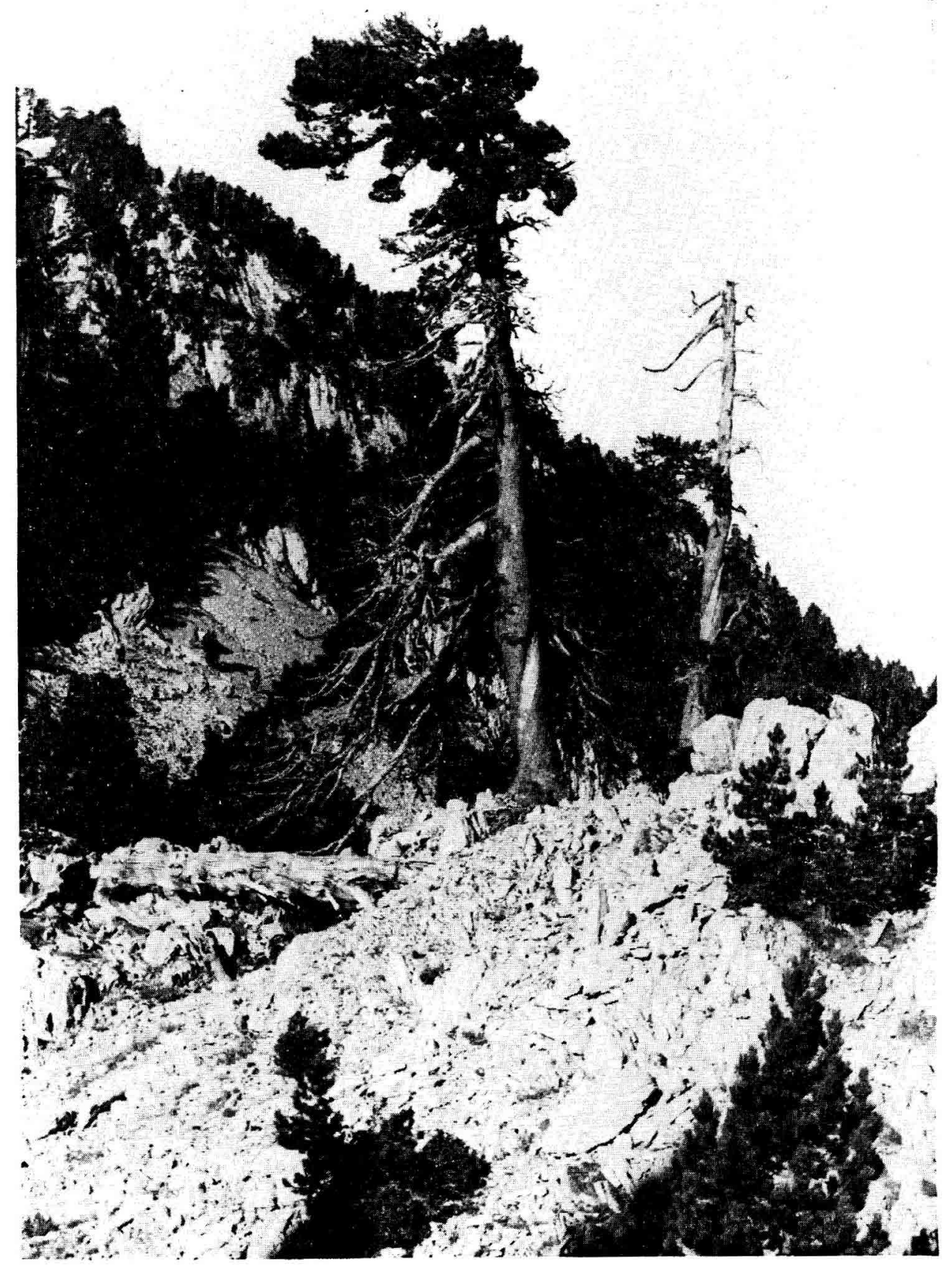




\section{Origine et évolution de la pineraie de Pints ancinata aux Pyrénées}

L'existence de deux populations reliques dans les Monts Jbériques, au-delà de la vasie plaine de l'Ebre, prouve que les Pyrénées ne constituent pas unc conquête récente du pin à crochets. Divers gisements de macrorestes indiquent qu'un pin très proche du pin de montagne existe en Europe dès le Tertiaire. Avant les bouleversements climatiques du Quatemaire, ce pin peuple probablement la zone élevée des montagnes beaucoup plus massivement que de nos jours. Par la suite, les glaciations successives démantèlent l'espace forestier et aceentuent la hardiesse du relief pyrénéen. Des discontinuités physiographiques engendrées résulte un isolement des divers îlots de pin à crochets qui évoluent au Postglaciaire en des types autonomes, surtout dans les sites marginaux.

Ainsi, la population extrême-occidentale d'Anie, et celle de la tourbière de Pinel. présentent-clles aujourd'hui un polymorphisme relativement faible que l'on peut interpréter comme une variabilité génétique réduite. Sans nécessairement invoquer ici des phénomènes de dérive génétique, on conçoit aisément que ces populations marginales évoluent pour leur propre compte sous la pression sélective du milicu, ne bénéficiant pratiquement pas d'apports polliniques lointains contrairement aux populations internes de la chaîne. Entre le massif d'Anie et les pineraies centrales et orientales, l'écoulement des gènes se lerait de proche en proche et à sens unique, à la faveur du transport de pollen par les vents d'Ouest. Cette situation singulière de la pineraie d'Anie en fait une race locale bien typée qu'il convient de protéger lant pour son pool génique que pour les richesses floristiques et faunistiques qu'elle recele.

Les formations daltitude à Pinus uncinata constituent des pineraies sauvages dont la conservation à l'écart des artificialisations chroniques et des pollutions génétiques devient une nécessité pour nous-mêmes et pour les générations futures. Ne venons pas briser de façon irréversible une dynamique héritée de millénaires d'une histoire mouvementée encore si mal connue.

\section{Remerciements}

Mes plus vifs remerciements vont à :

- C. Disdoneterhi, directeur du C.B.E.A. de l'Université de Pazu, qui a alcepté de diriger ma thèse de 3 " cycle el de la publier in extenso dans Acra Biologica Montana. 1983, 2-3, 87-330 dédition du Centre Pyrénéen de Biologic et Anthropologie des Montagnes, 8, rue Gérard-(al, F 64000) Paul);

- Colette Biannad-Dacian, professeur an Laboratoire de Physiologie cellulaire végétale de Bordcaux I, et son équipe, pour les analyses terpéniques;

- Ph. Baradar, maître de recherches au Laboratoire d'Amélioration des Arbres forestiers de I'I.N.R.A.-Bordeaux, pour l'utilisation de la programmathèque et les analyses statistiques:

- M. Ark31\%, directeur de ce laboratoire, pour ses conseils et ses encouragements:

- L. Discrolx, ex-stagiaire E.N.I.T.E.F. à I'I.N.R.A.-Bordeaux, pour le traitement informatique des fichiers:

- A. Bauditire, professeur à l'Université Paul-Sabatier de Toulouse, el M. JacaMON, professeur à I'E.N.G.R.E.F., qui, avec P. BARADAT, ont bien voulu relire le manuscrit et me faire part de leurs remarques. 


\section{Sumntary \\ Contribution to the study of biological and bicchemical variability of mountain pine (Pinus uncinata Ram.) within Pyrenees}

The mountain pine (P'ints ancinata Ramond) shows a wide range of forms, at individual level as well as between populations. In western Pyrenees, the morphological hiatus is specially stressed between the sickly trees of the Anie calcareous massif and the trees of the Neouvielle granitic massif, much more vigorous. The comparison in situ of these two provenances is based on a biosystematic study concerning a lot of characteristics, either morphological ones (pollen, needle, cone, seed, seedling) or biochenical ones (terpenes of cortical tissues).

The covariance analysis within populations permits to exclude from the taxonomic study, on the one hand, the characteristics directly sensitive to the environment effects, on the other hand, the redundant data. The characteristics of cone, needle and terpenic pattern, after having expurgated age and vigor effects, in the sample-trees, are subjected to a discriminant analysis. This one stresses the divergence previously obtained with the separate study of the characteristics of the two populations.

The fiability of terpenic characteristics as genetic markers leads to consider Anie and Neouvielle as two distinct taxa of mountain pine. The relatively low phenotypic variability of the westerner one (Anie) is probably due to its marginal position with regard to the Pyrenean area of Pinus uncinata.

Regu le 16 juillet 1982.

Accepté le 25 juillet 1985.

\section{Références bibliographiques}

Aytug B., 1962. Diagnose des pollens de Pinus silvestris et Pinus uncinala des Pyrénées. Pollen et Spores, 4 (2), 283-296.

Bernard-Dagan C., Baradat Ph., 1977. Utilisation des terpènes comme outil en génétique forestière. In : Symposium C.E.E. sur la biochimie de l'arbre forestier. Bruxelles. Ed. Commission des Communatués Européennes, EUR. 5885, 4, 109-152.

Bonnet-Masimbert M., 1979. Populations et isoenzymes chez Pinus nigra. Bull. Soc. Bot., Fr., Actual. hot., 2, 67-78.

Cabidoche Y.M., 1979. Contribution à l'étude des sols de haute montagne. Thèse doctorat 3" cycle, Univ. Montpellier.

CANTEGREL R, 1980. Recherches préliminaires sur la biosystématique du pin à crochets des Pyrénées occidentales. D.E.A., Université de Pau et des Pays de l'Adour, $59 \mathrm{p}$.

CANTEgrel. R., 1980. Etude biosystématique de deux populations pyrénée'nnes de pin à crochets (Pinus uncinata Ramond). Mémoire E.N.I.T.E.F. - Université de Pau, 62 p.

Cantegrli. R., 1982. Essai de discrimination de deux populations pyrénéennes de pin à crochets (Pinus uncinata Ram.), Anie et Néouvielle. Thèse doctorat 3" cycle, Université de Palu et des Pays de l'Adour, 237 p. + annexes.

Chovard P., 1938. Le rayonnement solaire et ses rapports avec la végétation dans le massif pyrénéen de Néouvielle. Trav. lab. forest. de Toulouse, 6 (2), art. 2.

Creus J.Y., Puigdefabregas J., 1976. Climatologia historica y dendrocronologia del Pinus uncinata Ramond. Separata de Cuadernos de Investigacion, 2 (2).

DEBLONDE P., 1974. Etude et mise au point de tests biochimiques appliqués aut recherches sur la variabilité génétique des arbres forestiers. Mémoire E.N.I.T.E.F.-C.N.R.F.

Decaix G., Tarditu F., 1980. Etude écologique et définition des stations forestièrés en Capcir, Cerdagne ef Haut Conflent. Mémoire E.N.I.T.E.F., 71 p. + annexes. 
Dendaletche Cl., 1973. Ecologie et peuplement végétal des Pyrénées occidentales. Essai d'écologie montagnarde. Thèse doctorat d'Etat, Nantes, $661 \mathrm{p}$.

Dendaletche Cl., 1982. Guia de los Pirineos. Geologia. Ecologia. Biologia. Omega Ed., Barcelona, $790 \mathrm{p}$.

Flous F., 1933. Les pins montagnards et subalpins des Pyrénées. Bull. Soc. Hist. Nat. Toulouse (65), 299-308.

Gaussen H., 1923, 1925, 1927. Le Pin à crochets dans les Pyrénées. Bull. Suc. Hist. Nat. Toulouse, LI : 581-600, LIII : 150-159, LV : 208-230.

Gaussen H., 1926. Végétation de la moitié orientale des Pyrénées. Sol. Climat. Végétation. Lechevalicr Ed., Paris, 560 p., 2 cartes hors-texte.

JALUT G., 1974. Evolution de la végétation et variations climatiques durant les quinze derniers millénaires dans lextrémité orientale des Pyrénées. Thèse doctorat d'Etat, Toulouse, $176 \mathrm{p}$.

Morelet M., 1979. Sur le dépérissement de Pinus uncinata dans la réserve du Néouvielle. Doc. C.N.R.F., Nancy.

Tanasescu N., 1939. Etude biométrique sur les cônes du Pin à crochets (Pinus uncinata Ramond). Bull. Facult. Agro. Chisinau, 2.

Tomassone R., 1963. Application des fonctions discriminantes à des problèmes biométriques. Ann. E.N.E.F., 20 (4), 583-617. 